Portland State University

PDXScholar

Winter 3-12-2018

\title{
Relationship between Anisotropy and Dispersive Stress in Wind Plants with Variable Spacing
}

Tamara S. Dib

Portland State University

Follow this and additional works at: https://pdxscholar.library.pdx.edu/open_access_etds

Part of the Mechanical Engineering Commons

Let us know how access to this document benefits you.

\section{Recommended Citation}

Dib, Tamara S., "Relationship between Anisotropy and Dispersive Stress in Wind Plants with Variable Spacing" (2018). Dissertations and Theses. Paper 4335.

https://doi.org/10.15760/etd.6228

This Thesis is brought to you for free and open access. It has been accepted for inclusion in Dissertations and Theses by an authorized administrator of PDXScholar. Please contact us if we can make this document more accessible: pdxscholar@pdx.edu. 
Relationship between Anisotropy and Dispersive Stress in Wind Plants with Variable Spacing

by

Tamara S. Dib

A thesis submitted in partial fulfillment of the requirements for the degree of

\author{
Master of Science \\ in \\ Mechanical Engineering
}

Thesis Committee:

Raúl Bayoán Cal, Chair

Elliott Gall

Derek Tretheway

Portland State University

2018 


\begin{abstract}
Large eddy simulations are considered for wind plants with varied spanwise and streamwise spacing. Data from five different configurations of staggered and aligned LES wind turbine arrays with a neutrally stratified atmospheric boundary layer are employed for analysis. Fields are analyzed by evaluating the anisotropy stress invariants based on the Reynolds shear stresses and dispersive stress tensor. The relationship between quantities are drawn as a function of the wind plant packing. Reynolds stresses and dispersive stresses are investigated alongside a domain altered version of the second and third scalar invariants, $\xi, \eta$, as well as the combination of the two invariants described by the function $F=1-27 \eta^{2}+54 \xi^{3} . F$ is a measure of the approach to either a two-component turbulence $(F=1)$ or an isotropic turbulence $(F=0)$. The invariant $\eta$ describes the degree of anisotropy while $\xi$ describes the characteristic shape. For the purposes of this study, the LES data is analyzed to understand the effects of canopy density on anisotropy and dispersive stresses, adding further insight and detail for future modeling techniques.
\end{abstract}




\section{Acknowledgements}

First and foremost, I would like to thank my advisor Raúl Bayoán Cal for his enthusiasm and motivation in my pursuit for continuing my education. I am grateful to Naseem Ali for his patience and guidance with this project as well as other projects. I would also like to express gratitude to Gerard Cortina and Marc Calaf for their assistance and access to the LES data used in this study. Finally, my family deserves huge credit on me getting this far with their continuous support in my intellectual journey. 


\section{Contents}

Abstract

Acknowledgements $\quad$ ii

List of Tables $\quad$ v

List of Figures $\quad$ vi

Nomenclature $\quad$ ix

Chapter 1 Introduction and Motivation 1

$\begin{array}{lll}\text { Chapter } 2 & \text { Theory } & 7\end{array}$

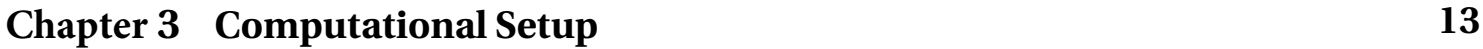

Chapter 4 Results: Time-Averaged Statistics and Chapter $\quad 18$

Chapter 5 Results: Subdomain Statistics $\quad 26$

$\begin{array}{lll}\text { Chapter } 6 & \text { Wake Analysis } & 38\end{array}$

$\begin{array}{lll}\text { Chapter } 7 & \text { Scatterplots } & 46\end{array}$

$\begin{array}{lll}\text { Chapter } 8 \text { Conclusion } & 54\end{array}$ 
Chapter 9 Future Work 58

Bibliography 


\section{List of Tables}

2.1 The Different States of Turbulence . . . . . . . . . . . . . . . . . . 11

3.1 Wind Farm Array Configurations $\ldots \ldots \ldots \ldots \ldots$

4.1 Subdomains in terms of streamwise distance $(x / D)$ from the entry into the canopy for all cases. . . . . . . . . . . . . . . . . . . . . . . 24 


\section{List of Figures}

2.1 The anisotropy invariant map, AIM [3] . . . . . . . . . . . . . 12

3.1 Drawing of the numerical domain . . . . . . . . . . . 15

3.2 Drawing of turbine array spacing and critical turbine components location with $\mathrm{HH}=$ hub-height, $\mathrm{BT}=$ bottom rotor tip, and $\mathrm{TT}=$ top rotor

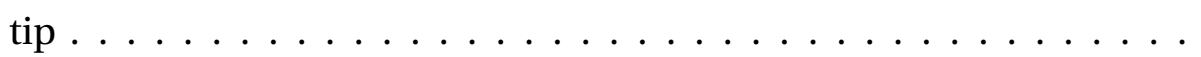

4.1 The normalized mean wind velocity, $\bar{u} / U$, along the streamwise at hub-height for all 5 cases in the turbine domain. The cases with increased spacing in the spanwise, $S_{x} \times 2 S_{z}$ and $2 S_{x} \times 2 S_{z}$, have the overall highest mean velocity magnitudes. . . . . . . . . . . . .

4.2 The normalized Reynolds shear stress, $\overline{u^{\prime} v^{\prime}} / U^{2}$, at hub-height for all 5 cases. Increasing the streamwise spacing, as seen in $2 S_{x} \times S_{z}$ and $2 S_{x} \times$ $2 S_{z}$, creates distinct breaks in Reynolds shear stress between each row implying that the Reynolds shear stress has less impact on subsequent turbines downstream for these cases. . . . . . . . . . . . . 22

4.3 The normalized turbulence kinetic energy, $\frac{1}{2}\left(\overline{u^{\prime 2}}+\overline{v^{\prime 2}}+\overline{w^{\prime 2}}\right) / U^{2}$, at hub height for all 5 cases. TKE is highest further downstream in the domain and is particularly pronounced in the two cases with the least spacing in both directions, $S_{x} \times S_{z}$ and $S_{x} \times S_{z}(S t) \ldots \ldots \ldots$ 
5.1 Normalized spatially averaged Reynolds stress in developing, fully developed and relaxed subdomain. $\left\langle\overline{u^{\prime} u^{\prime}}\right\rangle / U^{2}$ has the greatest magnitude among the three stresses. . . . . . . . . . . . . .

5.2 Spatially averaged dispersive stress in developing, fully developed and relaxed subdomain. Stresses follow a parabolic trend with the peak stress at hub-height. Developing subdomain has the highest stress, with the relaxed region with the lowest stress magnitude. . . . . . . . . 34

5.3 Spatially averaged invariants in the developing, fully developed and relaxed subdomains. Cases with the least amount of spacing between turbines, $S_{x} \times S_{z}$ and $S_{x} \times S_{z}$ (St), exhibit the most anisotropic behavior. 37

6.1 Spatially averaged Reynolds stress in the near and far wake. The near wake contains the highest contribution from the stresses. . . . . . . 40

6.2 Spatially averaged dispersive stress in the near and far wake. The momentum induced by spatial variations is highly influenced by the near

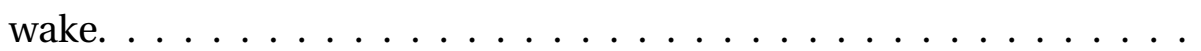

6.3 Spatially averaged invariants in the near and far wake. In both wake locations, the staggered case is the most isotropic, whereas the most spaced $2 S_{x} \times 2 S_{z}$ is the most anisotropic. In the near wake, there is a spike in anisotropic turbulence at the top rotor tip. This spike decays significantly in the far wake. $\ldots \ldots \ldots \ldots \ldots$ 
7.1 Reynolds stresses in the near wake in the fully developed regime for case $S_{x} \times S_{z}$. There is a linear correlation between the magnitude of Reynolds normal streamwise and shear stress and anisotropy. . . . . .

7.2 Dispersive normal streamwise stress vs. invariants in the near wake of the fully developed regime for $S_{x} \times S_{z}, 2 S_{x} \times 2 S_{z}, S_{x} \times S_{z}$ (St). The color map only includes the swept rotor area, whereas the black dots represent below the bottom rotor tip and above the rotor tip. . . . .

7.3 Dispersive normal spanwise stress vs. invariants in the near wake of the fully developed regime for $S_{x} \times S_{z}, 2 S_{x} \times 2 S_{z}, S_{x} \times S_{z}(\mathrm{St})$. The color map only includes the swept rotor area, whereas the black dots represent below the bottom rotor tip and above the rotor tip. . . . . . . .

7.4 Dispersive shear stress vs. invariants in the near wake of the fully developed regime for $S_{x} \times S_{z}, 2 S_{x} \times 2 S_{z}, S_{x} \times S_{z}$ (St). The color map only includes the swept rotor area, whereas the black dots represent below the bottom rotor tip and above the rotor tip. Increased spacing results in an overall higher dispersive stress as well as an increase in anisotropy between the bottom rotor tip and hub. . . . . . . . . . . 


\section{Nomenclature}

$\begin{array}{ll}D & \text { Diameter of the rotor } \\ a_{i j} & \text { Reynolds stress tensor } \\ b_{i j} & \text { Normalized Reynolds stress tensor } \\ \langle. .\rangle & \text { Spatial average } \\ \bar{u} & \text { Time-averaged velocity } \\ t & \text { Time, s } \\ U & \text { Upstream velocity for normalizing } \\ f_{x} & \text { Time averaged thrust force }\end{array}$




\section{Chapter 1}

\section{Introduction and Motivation}

An increase in economic growth and demand for energy production across the globe has resulted in more opportunities in renewable energy [27]. Initiatives such as the Kyoto Protocol and the Paris Agreement indicate a world-wide recognition to combat climate change. One of the key objectives for the Paris Agreement is to significantly lower green-house gas emissions, but in order for this to be successful, realizing energy efficiencies and renewable energy implementations are crucial [13]. Increasing energy demands and emission reduction goals will necessitate a diverse energy market that relies on energy efficiency and renewable energy production [15]. Developing countries account for $80 \%$ of the world's population, but only $30 \%$ of the world's consumption of energy [19]. An undiversified energy market in these countries is im-

practical with increased demand. Advancements in renewable energy such as wind energy have been found to not only help mitigate climate change, but reduce health costs (from air pollution) and obtain energy independence [19].

Although currently complex, wind energy has been around for roughly 3000 years. Originally, wind energy was converted to mechanical power to pump water and grind grain. In the early 1970s, wind energy began to be used for electricity generation [1]. Wind energy costs have dropped by one-sixth since the 1980s as a result of improved 
technology. In the 1990s, wind energy was the fastest growing energy technology with most installations and developments in Europe. Wind turbine technology is consistently being developing and improved as power capacity has increased from $67 \mathrm{~kW}$ in 1988 to around $1650 \mathrm{~kW}$ in 2003 [1].

For continuous growth in wind energy production, larger wind farms, both on and offshore, are required [20]. For smaller turbine arrays, minimum spacing between turbines is desirable in order to prevent fatigue loads from wakes in turbines directly downstream of other turbines. Wind farms are considered infinite once the array length exceeds the height of the atmospheric boundary layer by over an order of magnitude [20]. An infinite array is an idealized setting for most computational studies, but realistic arrays are in fact finite. Large arrays effect the ABL differently than finite arrays. Minimum spacing in finite arrays increases the effective surface roughness in the ABL and decreases effective wind velocity at hub height and ground. For larger arrays, efficiency decreases from interactions between the total drag of the turbines and $\mathrm{ABL}$, making it crucial to constantly explore turbine array efficiency in both finite and infinite arrays [6]. Spatial variations of wind turbine placements in arrays can highly affect both performance and economics [14]. An increased understanding of spatial variability will provide increased energy production and economic savings when implemented into a turbine array.

To better understand the effect of spatial variations in wind farms, it is important to look at dispersive stresses. Calculating double averaged momentum equations for both the temporal and spatial domain result in dispersive stresses [22]. In the past, dispersive stresses were ignored, but the temporally and spatially averaged momentum equations have introduced notable terms for dispersive fluxes and wake produc- 
tion [25].

Dispersive stresses have been found to be more severe in sparse canopies than dense canopies. In a study by [23], dispersive stresses in dense canopies were found to be insignificant across the canopy equating to less than $5 \%$ of the Reynolds stress. In sparse canopies high dispersive stresses, up to $35 \%$ of the Reynolds stress were found in the bottom layers of the canopy. A study was conducted on the importance and dominance of dispersive stresses in open-channel flow over rough beds [22]. Gravelbed rivers are a prime example of flow over irregular rough beds as roughness elements often extend up to the surface. Since the beds are spatially heterogeneous, it was found that only temporally averaged momentum equations were not sufficient for describing the flow as double averaged equations included the necessary drag and form-induced momentum fluxes.

In an upstream edge (entry region) of a forest patch canopy where in-canopy velocities are large, the magnitude of the normal dispersive stresses were significantly larger than the normal Reynolds stresses. The dispersive stresses influenced the rate at which momentum entered the canopy, but further downstream the dispersive stresses decreased remarkably as the ABL developed in the canopy [21]. In a study with arrays of urban-like cubical obstacles, discoveries of high significance in dispersive stresses within the cubes were found to have a higher dominance in Reynolds stress above the cubes [8]. Dispersive stresses were used to study flow resistance due to vegetation; since there was spatial variation among the plants, spatial variation induced stresses were necessary for a model description of the flow [26]. For turbulent flow that contains spatial heterogeneity these past studies confirm the importance of including dispersive stresses in analysis. 
In many engineering applications, understanding the amount and type of anisotropy in turbulence is important for the closure of Reynolds-averaged NavierStokes (RANS) equations when modeling turbulent flows based on turbulent statistical models [5]. Lumley developed the anisotropy invariant map (AIM) often referred to as Lumley or Turbulence triangle through the implementation of the anisotropy stress tensor invariants [18]. This theory of Lumley is used to describe the time mean invariants, where the Lumley Triangle is a domain where all Reynolds stress invariants lie [9]. The eigenvalue decomposition of the Reynolds stress tensor are correlated to spherical shapes as visual representations for anisotropy of turbulence [14]. These analyses by Lumley provide a useful tool to measure the degree of anisotropy in turbulence often used for developing turbulent models [2].

In a study on the effect of thermal stratification on flow structure, [2] found a direct correlation between anisotropy and TKE production, with anisotropy increasing as TKE increased. The aim of the study was to understand the time-varying interaction between wind turbines and turbulent atmospheric flow by comparing stable, unstable, and neutral stratification cases. The study concluded that turbulent mixing from buoyancy was directly correlated to anisotropy and energy distribution while unstable stratified flow was found to be more isotropic than stable and neutral stratified flow. Another study on the anisotropy of the Reynolds stress tensor with wind turbine arrays, focused on the anisotropy of the turbine wake from counter clockwise rotation versus clockwise rotation [14]. Results indicated that anisotropy of turbine array should not be ignored since the flux of kinetic energy and production of turbulent kinetic energy were also directly associated with the anisotropy stress invariants.

Another investigation on anisotropy found that polymer drag reduction is related 
to increasing anisotropy in the near-wall region [16]. This study was continued on polymer drag reduction using the anisotropy invariant map as found by Lumley. It was found that in order to achieve higher drag reduction and energy savings, near wall fluctuations need to be primarily one-component in the mean flow direction [12]. The anisotropy invariant map was utilized based on the values of the Reynolds stress tensor to analyze the gap between the local turbulence and the 3D isotropic state in a mixing tank stirred with a Rushton turbine. It was determined that high levels of anisotropy occurred not only near the impeller but near the vessel bottom and within the circulation loops [10].

When analyzing wind turbines, it is often beneficial to study the wakes caused by the turbines themselves. Wind turbine wakes are most commonly described with the near wake and far wake. The near wake are regions 2-4 rotor diameters downstream of the turbines, where the flow is heavily influenced by the rotor blades. In this region, there is an increase in turbulence and velocity deficit caused by energy extraction and vortices created by the mean flow at the tip of the rotor blades [4]. This energy extraction causes a relaxation in pressure gradients and the wake width increases as the air moves downstream from the rotor. Once past the near wake, the velocity begins to recover as fluid mixing takes over and the flow location becomes the far wake. The far wake is further downstream where the location typically varies depending on the characteristics of the flow. In the far wake, both the velocity deficit and extra turbulence begin to decline as tip vortices decay [4] .

It is common practice for wind farms to be analyzed through computational fluid dynamics, especially since the rise of supercomputers. In academia, Direct Numerical Simulation (DNS) and Large Eddy Simulation (LES) have been most prominently 
used. DNS incorporates all scales, including large energy-containing (integral scales) to the smaller dissipative scales (Kolmogoroff). DNS requires a large number of grid points which results in a costly computation. LES is expensive, but it can be seen as a more cost efficient middle ground. LES is three dimensional and time dependent, but explicitly resolves larger, more energetic and often more important scales. It uses a secondary filter, or subgrid-scale (SGS) model, for the smaller scales which are more universal and easier to model [11].

In this work, five available data sets from a LES wind array turbine study were examined to provide further insight on the effect of spatial variability in canopies. Since wind turbine arrays can be regarded as canopies, spatial variability should not be neglected in discussing the flow. For this research, spatial variation in large finite wind turbine arrays will be compared to horizontally averaged anisotropy invariants, dispersive stresses, and Reynolds stresses. 


\section{Chapter 2}

\section{Theory}

\subsubsection{Governing Equations}

Considering a turbulent boundary layer forced in the $\mathrm{x}$ direction, the resulting steady Reynolds averaged momentum and continuity equations at a point for a wind turbine canopy are

$$
\begin{gathered}
\overline{u_{j}} \frac{\partial \overline{u_{i}}}{\partial x_{j}}=-\frac{1}{\rho} \frac{\partial \bar{p}}{\partial x_{i}}-\frac{\partial \overline{u_{i}^{\prime} u_{j}^{\prime}}}{\partial x_{j}}+v \frac{\partial^{2} \overline{u_{i}}}{\partial x_{j}^{2}}+\overline{f_{x}} \\
\frac{\partial u_{k}}{\partial x_{k}}=0
\end{gathered}
$$

where $x_{i}$ represent direction in the streamwise, wall-normal and transverse, respectively, $u_{i}$ are the instantaneous velocity components along $x_{i}, p$ is the pressure, $\rho$ is density, $\overline{u_{i}^{\prime} u_{j}^{\prime}}$ is the turbulent Reynolds stress, $v\left(\frac{\partial^{2} \overline{u_{i}}}{\partial x_{j}^{2}}\right)$ is the viscous stress and $\overline{f_{x}}$ is the time averaged thrust force (included from the effect of wind turbines). The thrust force has a subscript $x$ because the force acts primarily in the streamwise direction.

Instantaneous velocities decomposed into a mean and its fluctuations results with,

$$
u=\bar{u}+u^{\prime}
$$


with $u$ as the instantaneous velocity component, $\bar{u}$ denoting the time averaged velocity component, and $u^{\prime}$ as the temporal fluctuation. Correlating the temporal fluctuation terms results with the symmetric second order turbulent Reynolds stress tensor, $\overline{u_{i}^{\prime} u_{j}^{\prime}}$.

\subsubsection{Dispersive Stresses}

The spatial averaging of the momentum equation produces the dispersive stress terms that describe spatial variation in turbulence. To obtain dispersive stresses, also referred to as form-induced stresses, time averaged velocities are further decomposed into spatial means [21]. The Reynolds stresses are the result of temporal decomposition and averaging, whereas dispersive stresses are the result of decomposing the velocity variable and spatial averaging of the momentum equation.

The time averaged velocity term decomposed from the spatial mean results with,

$$
\bar{u}=\langle\bar{u}\rangle+\bar{u}^{\prime \prime}
$$

where $\langle.$.$\rangle represents the spatial average, and spatial fluctuation is shown as double$ prime. This results with the following spatial averaged dispersive stress denoted as $\left\langle\overline{u_{i}^{\prime \prime} u_{J}^{\prime \prime}}\right\rangle$.

Applying time and spatial averaging results in the following spatially-averaged momentum equation,

$$
\left\langle u_{j}\right\rangle \frac{\partial\left\langle\overline{u_{i}}\right\rangle}{\partial x_{j}}=-\frac{1}{\rho} \frac{\partial\langle\bar{p}\rangle}{\partial x_{i}}-\frac{\partial\left\langle\overline{u_{i}^{\prime} u_{j}^{\prime}}\right\rangle}{\partial x_{j}}-\frac{\partial\left\langle\overline{u_{i}^{\prime \prime} u_{j}^{\prime \prime}}\right\rangle}{\partial x_{j}}+v \frac{\partial^{2}\left\langle\overline{u_{i}}\right\rangle}{\partial x_{j}^{2}}+\left\langle\overline{f_{x}}\right\rangle .
$$

The momentum equation now includes the dispersive stress, $\left\langle\overline{u_{i}^{\prime \prime} u_{j}^{\prime \prime}}\right\rangle$, as well as the Reynolds stress and viscous stress. The dispersive stresses represent momentum flux 
caused by point to point spatial correlations in time averaged velocity fields. It provides extra physical processes that occur in the canopy as well as more information on the mean and turbulent kinetic energy balance [17].

\subsubsection{Anisotropy Stress Tensor}

The second order Reynolds stress tensor, $\overline{u_{i}^{\prime} u_{j}^{\prime}}$, is significant in turbulence because it describes the balance of stresses at any given location. This tensor is symmetric and shown as

$$
\overline{u_{i}^{\prime} u_{j}^{\prime}}=\left[\begin{array}{ccc}
\overline{u^{\prime 2}} & -\overline{u^{\prime} v^{\prime}} & -\overline{u^{\prime} w^{\prime}} \\
-\overline{u^{\prime} v^{\prime}} & \overline{v^{\prime 2}} & -\overline{v^{\prime} w^{\prime}} \\
-\overline{u^{\prime} w^{\prime}} & -\overline{v^{\prime} w^{\prime}} & \overline{w^{\prime 2}}
\end{array}\right] .
$$

The terms on the diagonal are normal stress whilst the rest of the terms are shear stresses. The summation of the diagonal terms is called the trace.

Given any second order tensor, $S$, its trace, $\operatorname{Tr}(S)$, can be used to decompose the tensor into an isotropic part and a deviatoric or anisotropic part [24],

$$
S=S^{i s o}+S^{\text {aniso }}=\frac{\operatorname{Tr}(S)}{3} I+S^{\text {aniso }} .
$$

Since the Kronecker delta term, $\delta_{i j}=\mathrm{I}$ and $s_{i i}=\operatorname{Tr}(S)$ with $S^{\text {aniso }}=S^{\prime}$

$$
S=\frac{1}{3} s_{i i} \delta_{i j}+S^{\prime}
$$

Using the second order Reynolds Stress tensor $\left(\overline{u_{i}^{\prime} u_{j}^{\prime}}\right)$ to quantify the anisotropy of turbulence, the Reynolds Stress anisotropy tensor $a_{i j}$ developed by Rotta is defined as, 


$$
a_{i j}=\overline{u_{i}^{\prime} u_{j}^{\prime}}-\frac{2}{3} k \delta_{i j}
$$

where $k=\frac{1}{2}\left(\overline{u^{\prime 2}}+\overline{v^{\prime 2}}+\overline{w^{\prime 2}}\right)$ is the turbulence kinetic energy and $2 k$ is the trace of $\overline{u_{i}^{\prime} u_{j}^{\prime}}$ [14]. This tensor is used to describe the preferential directions of the turbulent stress field. The anisotropic tensor is normalized by $2 k$ to create the zero trace tensor denoted by

$$
b_{i j}=\frac{\overline{u_{i}^{\prime} u_{j}^{\prime}}}{\overline{u_{k}^{\prime} u_{k}^{\prime}}}-\frac{1}{3} \delta_{i j} .
$$

For a symmetric second order tensor, there are three scalar principal invariants, $I, I I$, and $I I I$, whose values are the same in any coordinate system. Since, the anisotropy tensor has zero trace, $I=b_{i i}=0$, only the second and third invariants are used to visualize anisotropy. In order to see the nonlinear behavior in the return to isotropy more clearly and ['to examine the trajectories of the return to isotropy of homogeneous turbulence, variables $\eta$ and $\xi$ were introduced to describe the anisotropic stress invariants. The domain is altered where,

$$
\begin{gathered}
\xi^{3}=\frac{I I I}{2}=\frac{b_{i j} b_{j k} b_{k i}}{6}, \\
\eta^{2}=\frac{I I}{3}=\frac{b_{i j} b_{j i}}{6},
\end{gathered}
$$

where $\eta$ describes the degree of anisotropy and $\xi$ describes the characteristic shape. The squared $\eta$ scalar implies consistent positive values, but for $\xi$ a positive value or negative value indicates the flow is dominated by one-component turbulence or 


\begin{tabular}{ccc}
\hline \hline State of Turbulence & Invariants & Shape of Spheroid \\
\hline Isotropic & $\xi=\eta=0$ & Sphere \\
Two-component axisymmetric & $\xi=-\frac{1}{6}, \eta=\frac{1}{6}$ & Disk \\
One-component & $\xi=\eta=\frac{1}{3}$ & Line \\
Axisymmetric, one large eigenvalue & $\xi=\eta$ & Prolate spheroid \\
Axisymmetric, one small eigenvalue & $-\xi=\eta$ & Oblate spheroid \\
Two-component & $\eta=\left(\frac{1}{27}+2 \xi^{3}\right)^{1 / 2}$ & Ellipse \\
\hline
\end{tabular}

Table 2.1: The Different States of Turbulence

two-component turbulence respectively. These invariants are often depicted in an anisotropy invariant map (AIM), where the second invariant is plotted as a function of the third invariant, often referred to as the Lumley triangle shown in Figure 2.1 [5]. The borders within the map describe the different states of the turbulent stress tensor [29]. The two curves on the map portray axisymmetric turbulence, where the right and left curves represent axisymmetric expansion and contraction, respectively [12]. At the origin, the state is isotropic. It is common in studies to assume smaller scales are isotropic, but larger energetic scales are rarely isotropic. In wakes, such as turbine wakes, axisymmetric wakes are most prevalent [14]. The shape of the spheroids are either oblate or prolate spheroids of anisotropic turbulence. These shapes are outlined in table 2.1 .

Another function to describe anisotropy that combines the two invariants is

$$
F=1-27 \eta^{2}+54 \xi^{3}
$$

where turbulence approaches a two-component state at $F=0$ or a three-component isotropic state at $F=1[31]$. 


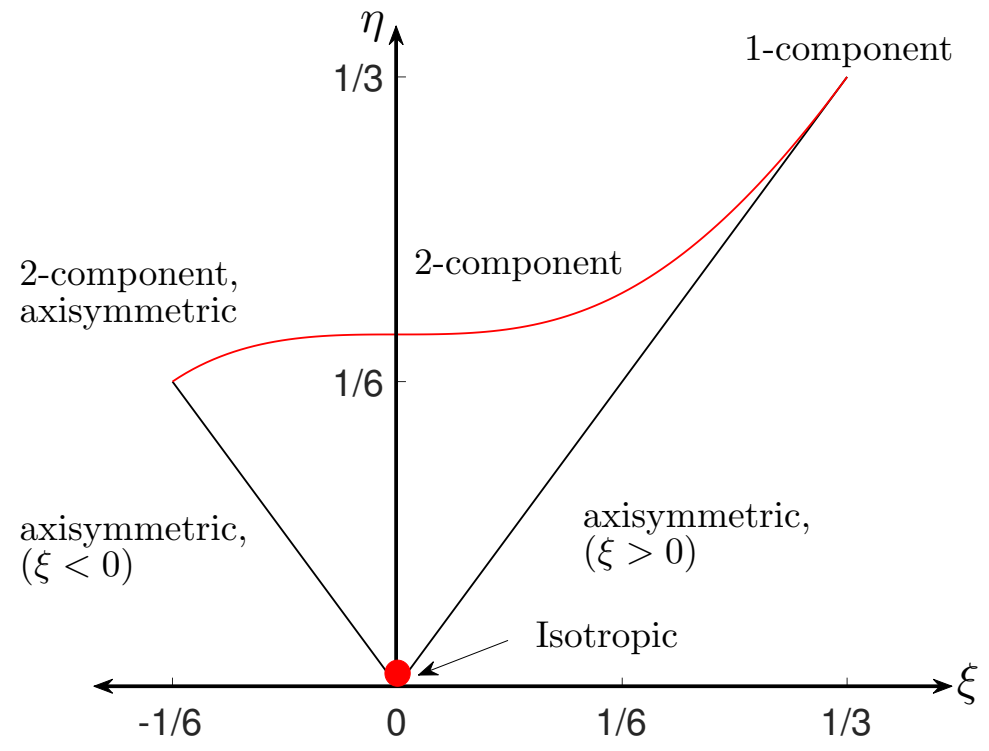

Figure 2.1: The anisotropy invariant map, AIM [3] 


\section{Chapter 3}

\section{Computational Setup}

\subsubsection{LES}

The study consists of five large wind turbine arrays with a neutrally stratified atmospheric boundary layer (ABL) rather than a thermally stratified ABL. A neutral ABL is representative for offshore and high wind speeds. The large eddy simulation equations are based off the following integrated filtered Navier Stokes and continuity equations,

$$
\begin{aligned}
\frac{\partial \tilde{u}_{i}}{\partial t}+\tilde{u}_{j}\left(\frac{\partial \tilde{u}_{i}}{\partial x_{j}}-\frac{\partial \tilde{u}_{j}}{\partial x_{i}}\right) & =-\frac{1}{\rho} \frac{\partial p}{\partial x_{i}}-\frac{\partial \tilde{\tau}_{i j}}{\partial x_{j}}+f_{i}^{w t}, \\
\frac{\partial \tilde{u}_{i}}{\partial x_{i}} & =0 .
\end{aligned}
$$

All variables are normalized by the surface friction velocity, $u_{*}$, and the boundary layer height, $y_{i}$. Drag and lift forces are calculated using a traditional actuator-disk with rotation model (ADM-R), where $f_{i}^{w t}$ represents the force imposed by the presence of the wind turbines. The tilde (...) represents the LES filtering operation at the grid size $\triangle$. The driving force in the ABL is the geostophic wind, with the flow driven by an imposed constant pressure gradient, such that $\frac{1}{\rho} \frac{\partial p_{\infty}}{\partial x}=1$ in the streamwise direction. This force is approximately equivalent to a wind forcing of close to $10 \mathrm{~m} / \mathrm{s}$ at the top of the 
domain. $\tau_{i j}$ is the filtered subgrid-scale stress term and its trace $\left(\tau_{k k} / 3\right)$ is combined with the pressure term. The deviatric component of $\tau_{i j}$ is equal to $\tau_{i j}-\frac{1}{3} \delta_{i j} \tau_{k k}$ and is modeled using the Smagorinsky eddy viscosity subgrid scale model with a constant smagorinsky coefficient $C_{s}=0.16$ [30]. The numerical discretization uses a pseudospectral approach for periodic boundary conditions in the horizontal direction and a second-order finite differencing in the vertical direction. The model is time integrated, for a million time-steps, using a second order Adam Bashford scheme. The nonlinear convective terms are dealiazed using the $3 / 2$ rule and the algorithm is fully parallelized using the Message-Passing Interface (MPI). At the surface a non-slip condition is used for the vertical velocity and first grid point for horizontal velocities due to the staggered grid. At the bottom surface the shear stress is parametrized using the standard $\log$ (Monin-Obukhov) similarity law

$$
\tau_{i, 3}\left(x, z, y_{1}\right)=-\left[\frac{k \sqrt{\hat{\tilde{u}}_{1}^{2}+\hat{\tilde{u}}_{2}^{2}}}{\ln \left(y_{1} / y_{0}\right)+\psi\left(y_{1} / L\right)}\right]^{2} n_{i}
$$

Where $(\hat{\ldots})$ ) represents a secondary filtering operation with twice the grid size $(2 \triangle)$. The height of the first grid point is $y_{1}$ where the shear stress is imposed, $y_{i}$ is the initial inversion height and $y_{0}$ is the ground surface roughness where $y_{0}=10^{-4} y_{i}$. The unitary directional vector is $n_{i}=\hat{\tilde{u}}_{i}^{2} / \operatorname{sqr} t\left(\hat{\tilde{u}}_{1}^{2}+\hat{\tilde{u}}_{2}^{2}\right)$, where $i$ is any of the horizontal plane-parallel directions.

\subsubsection{LES Model Cases}

The study consists of five different wind farm configurations of a numerical domain of size $(32 \pi \times 1 \times \pi) y_{i}$ in the streamwise, vertical and spanwise direction, respectively $\left(y_{i}\right.$ is the boundary layer height of $1000 \mathrm{~m}$ ). The numerical grid contains $4096 \times 128 \times 128$ 

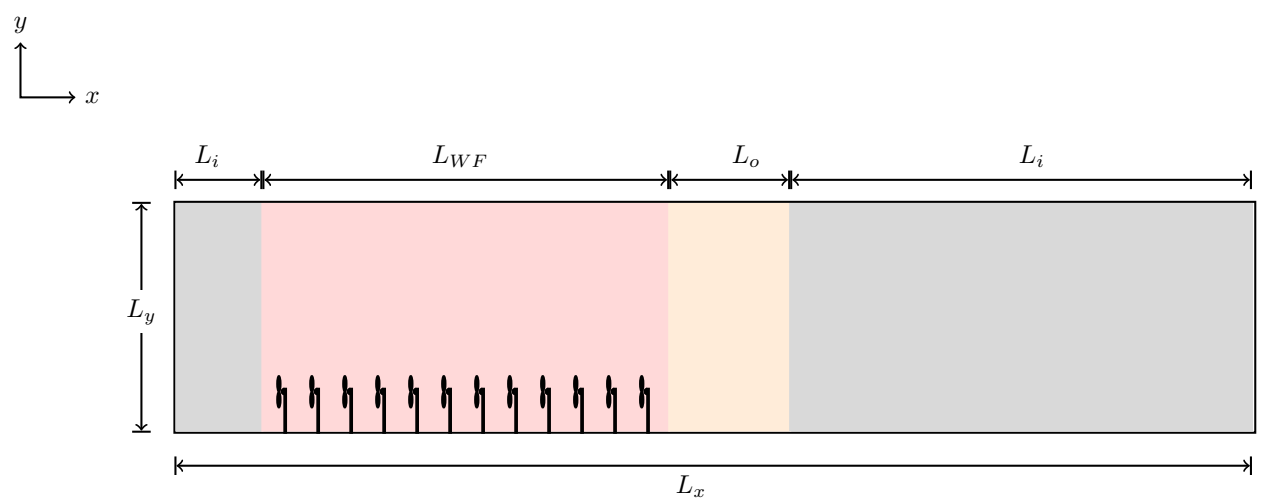

Figure 3.1: Drawing of the numerical domain

grid points with the highest resolution in the vertical direction. The total resolution is $24.5 m \times 7.85 m \times 24.5 m$, in the respective locations. To emulate a realistically large but finite wind farm, the turbines make up $18 \%$ of the streamwise domain in order for the flow to recover at the end of the domain. In the spanwise direction, it is periodic and infinite with the turbines occupying the entire space. Figure 3.1 is a simplified schematic of the domain with $L_{i}$ the section for the incoming flow, $L_{w f}$ is the domain with the wind turbines, $L_{o}$ the outlet section. Since the flow is periodic, $L_{i}$ is shown once again after $L_{o}$.

Each configuration varies in spacing for streamwise and spanwise directions. Figure 3.2a depicts the orientation for the spacing whereas figure 3.2b highlights some critical locations of a turbine. For this study, the top rotor tip has a vertical height of $155 \mathrm{~m}$, the hub height is $100 \mathrm{~m}$ high, and bottom rotor tip is $47 \mathrm{~m}$. For the normalized vertical numerical domain referenced in figures in later sections, the hub height is at $y / D=1$, top rotor tip is at $y / D=1.5$ and bottom rotor tip is at $y / D=0.5$. Table 3.1 shows all of the turbine spacing configurations for each case. The base turbine array configuration has a total of 24 turbines with 8 turbines in each row aligned with a spacing of 


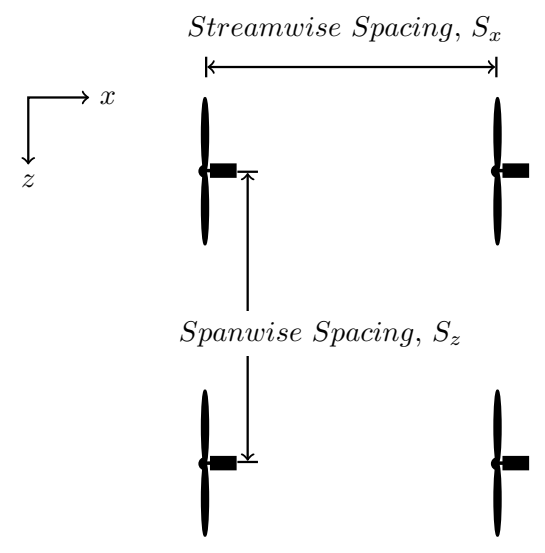

a.) Top View Drawing of Turbine Spacing

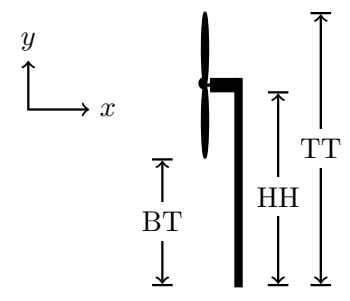

b.) Side View of Critical Turbine Components

Figure 3.2: Drawing of turbine array spacing and critical turbine components location with $\mathrm{HH}=$ hub-height, $\mathrm{BT}=$ bottom rotor tip, and $\mathrm{TT}=$ top rotor tip

$8 D$ in the streamwise and $4 D$ in the spanwise $\left(S_{x} \times S_{z}\right)$. All other cases will be represented off of the base case. The second case is $8 D$ in the streamwise with the spanwise doubled to $8 D\left(S_{x} \times 2 S_{z}\right)$. The third case is doubled in the stream wise spacing at $16 D$ with $4 D$ in the spanwise $\left(2 S_{x} \times S_{z}\right)$. The fourth case has spacing doubled in both the streamwise and spanwise direction, $16 D$ and $8 D$ respectively $\left(2 S_{x} \times 2 S_{z}\right)$. Lastly there is also a turbine configuration similar to the first case, but with a staggered alignment $\left(S_{x} \times S_{z}(\mathrm{St})\right)$ 


\begin{tabular}{cccc}
\hline \hline Case & Streamwise Spacing $\left(\mathrm{S}_{x}\right)$ & Spanwise Spacing $\left(\mathrm{S}_{z}\right)$ & Configuration \\
\hline $\mathrm{S}_{x} \times S_{z}$ & $8 \mathrm{D}$ & $4 \mathrm{D}$ & Aligned \\
$\mathrm{S}_{x} \times 2 S_{z}$ & $8 \mathrm{D}$ & $8 \mathrm{D}$ & Aligned \\
$2 \mathrm{~S}_{x} \times S_{z}$ & $16 \mathrm{D}$ & $4 \mathrm{D}$ & Aligned \\
$2 \mathrm{~S}_{x} \times 2 S_{z}$ & $16 \mathrm{D}$ & $8 \mathrm{D}$ & Aligned \\
$\mathrm{S}_{x} \times S_{z}(S t)$ & $8 \mathrm{D}$ & $4 \mathrm{D}$ & Staggered \\
\hline
\end{tabular}

Table 3.1: Wind Farm Array Configurations

The original intent of this study was to analyze the flow adjustment and flow equilibrium in causal effect of turbine arrangement. The high cost of LES studies limit them to academic and research use with most prior LES studies using infinite wind farms. By using finite-sized wind farms, the aim was to further understand three variables typically used in wind-energy models: the wind-farm induced effective roughness length, the wake-induced eddy viscosity, and the wake-expansion coefficient. Results from this study found that flow adjustment is mainly influenced by streamwise spacing with flow equilibrium achieved in the most dense configuration at row 18. For more information regarding this study reference [28]. For the purposes of this current paper, this study will be used to further understand the effects of canopy density on anisotropy and dispersive stresses. 


\section{Chapter 4}

\section{Results: Time-Averaged Statistics and Domain}

\subsubsection{Mean Velocity, Reynolds Shear Stress, and Turbulence Kinetic Energy}

Since this study is interested mainly in spatial heterogeneity within the canopy, only $25 \%$ of the streamwise numerical domain will be analyzed to focus on the volume directly before the turbines, the turbines themselves, and the outlet approximately 70$74 D$ downstream of the arrays. Figures 4.1, 4.2, and 4.3 are contours of the mean wind

velocity, $\bar{u}$, Reynolds shear stress, $\overline{u^{\prime} v^{\prime}}$, and turbulence kinetic energy $k=\frac{1}{2}\left(\overline{u^{\prime 2}}+\overline{v^{\prime 2}}+\right.$ $\left.\overline{w^{\prime 2}}\right)$, respectively at hub height for all five cases. The velocities are normalized by an unperturbed upstream velocity at hub height, $U$. The Reynolds shear stress and the turbulence kinetic energy are normalized by the square of the upstream velocity, $U^{2}$.

\section{Mean Velocity}

The mean velocities of the densest cases $S_{x} \times S_{z}$ and $S_{x} \times S_{z}$ (St) shown in figure 4.1 are lowest compared to the other cases throughout the domain. For both of these cases, the highest mean velocities are at the entry region $(x / D=0-16)$ of the canopy. The base case, $S_{x} \times S_{z}$, exhibits high velocity channels between the turbines up to $x / D=32$. The channeling continues past $x / D=32$, but with a $20 \%$ decline in magnitude. $S_{x} \times S_{z}$ also has acute lateral wake expansion that merges with its surrounding wakes, especially 
further downstream starting from $x / D=96$ and onwards.

The cases with the increased spanwise spacing, $S_{x} \times 2 S_{z}$ and $2 S_{x} \times 2 S_{z}$, also have high velocity channels, that extend further into the domain. $S_{x} \times 2 S_{z}$ has channels extending throughout the domain particularly at lateral distances between $y / D=16$ 32 and $y / D=48-64$. For case $2 S_{x} \times 2 S_{z}$, these begin to decay around $x / D=64$, where it reaches an observed fully developed state. These two cases have the overall highest magnitude in velocity among the rest. They both also exhibit the least amount of lateral wake interaction.

By increasing only the streamwise spacing, $2 S_{x} \times S_{z}$ has its highest velocities in the entry region $(x / D=0-24)$, then reaches a fully developed state at $x / D=100$. It also

has high velocity channels present throughout the domain, that are absent in the staggered case. The staggered case shows the most rapid deceleration with magnitudes decreasing around $20 \%$ from the entrance to $x / D=32$. The staggered nature of this case results in minimal wake interaction throughout the domain.

\section{Reynolds Shear Stress}

The shear stresses are the largest contributors to the mean kinetic energy budget, especially for flux and production, so it is important to observe these stresses as well as their vertical derivative. The normalized Reynolds shear stress at hub height for all five cases is shown in figure 4.2. The Reynolds shear stress is observed to be symmetric along the turbine axis for all cases. The turbines are the main factor for stress, and the stress continuously increases as the flow moves downstream from the entry to the exit region of the domain.

All cases except for the staggered case have streamwise channels of lesser magni- 

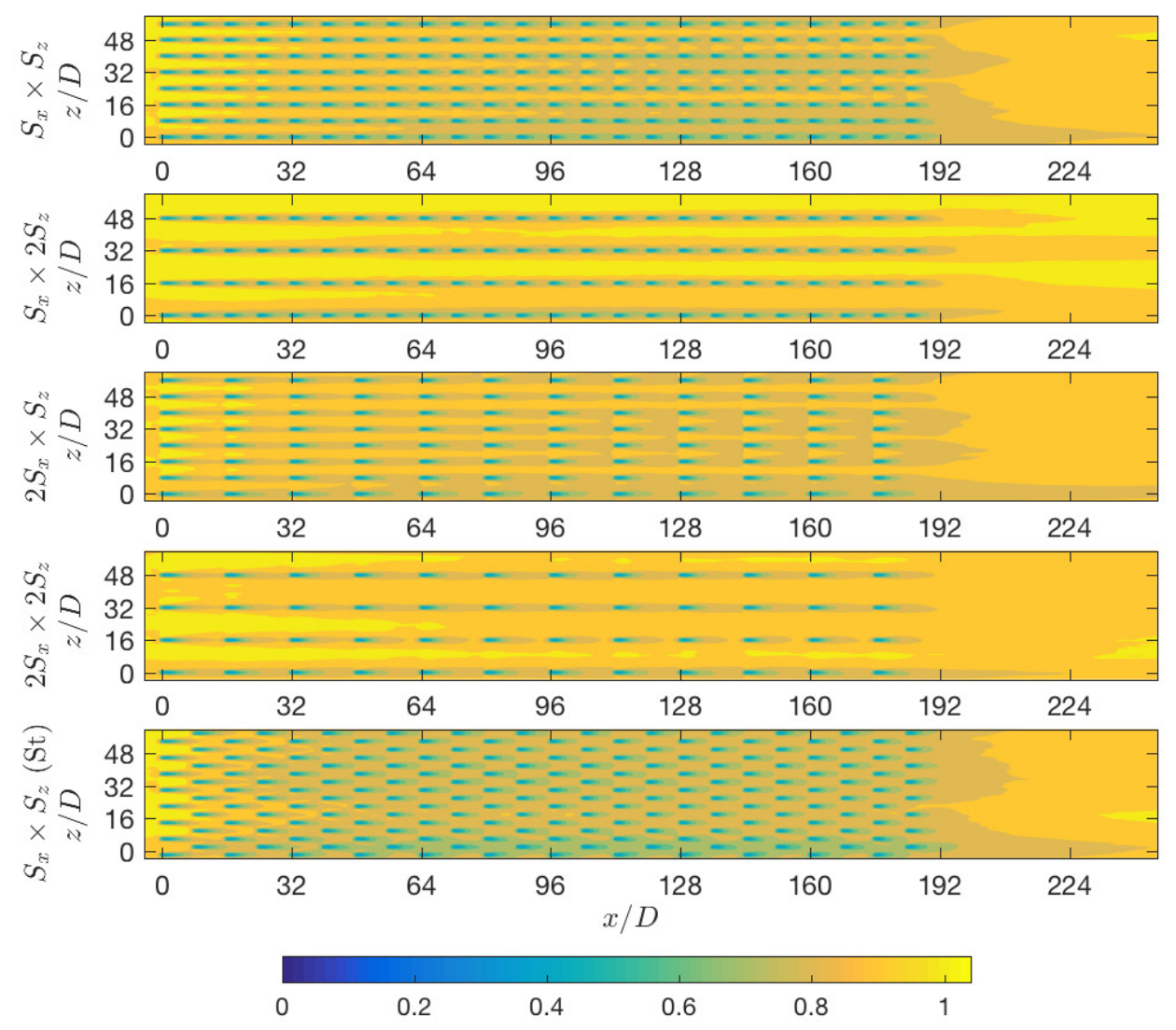

Figure 4.1: The normalized mean wind velocity, $\bar{u} / U$, along the streamwise at hubheight for all 5 cases in the turbine domain. The cases with increased spacing in the spanwise, $S_{x} \times 2 S_{z}$ and $2 S_{x} \times 2 S_{z}$, have the overall highest mean velocity magnitudes.

tude stresses between the turbines. Cases $S_{x} \times S_{z}$ and $S_{x} \times 2 S_{z}$ both have the highest magnitude of stress among the five cases, with stresses up to $14 \%$ larger. The first row in both of these cases have stresses $15 \%$ less than that of the preceding row then the stresses continue to increase downstream with values increasing $26 \%$ from row 1 to $x / D=128$.

Increasing the streamwise spacing, as seen in $2 S_{x} \times S_{z}$ and $2 S_{x} \times 2 S_{z}$, creates distinct 
breaks as the stress diffuses between each row implying that the stress has less impact on subsequent turbines downstream for these cases.

Increasing the spanwise spacing correlates to the stress channels continuing longer downstream past the last turbine row, whereas in the other cases Reynolds shear stress diffuses earlier in the domain. For $S_{x} \times 2 S_{z}$, the extension is approximately the end of the given domain, even further than $2 S_{x} \times 2 S_{z}$. The stress channels in $S_{x} \times S_{z}$ and $2 S_{x} \times S_{z}$, start mixing around $x / D=200$, but the cases with spanwise spacing, $S_{x} \times 2 S_{z}$ and $2 S_{x} \times 2 S_{z}$, do not start mixing until $x / D=224$.

The staggered cases does not exhibit this behavior, but has signs of lateral stress interaction and mixing throughout the domain.

\section{Turbulence Kinetic Energy}

Turbulence kinetic energy, TKE, shown in figure 4.3, increases as it develops downstream for all cases. In $S_{x} \times S_{z}$ and $S_{x} \times 2 S_{z}$, TKE increases up to $150 \%$ from the first row to last row. These two cases also have the overall highest TKE levels, up to $20 \%$ larger than the cases with an increase in streamwise spacing. This is because the wakes in these cases have less streamwise spacing for TKE to decay past the wake, resulting in a compounded kinetic energy further downstream past multiple wakes.

Increasing the spacing in both streamwise and spanwise directions results in more rapid decay of TKE past the last turbine row. Case $2 S_{x} \times 2 S_{z}$ shows full decay at $x / D=200$ and $S_{x} \times 2 S_{z}$ fully decays between $x / D=224-230$. Increasing only the streamwise spacing, $2 S_{x} \times S_{z}$, results with TKE not fully decaying in the given domain, but at $x / D=224$ approximately $80 \%$ of the domain shows signs of decay. For the other two cases, in particular $S_{x} \times S_{z}(S t)$, the TKE does not $100 \%$ fully decay in the given domain. Even 

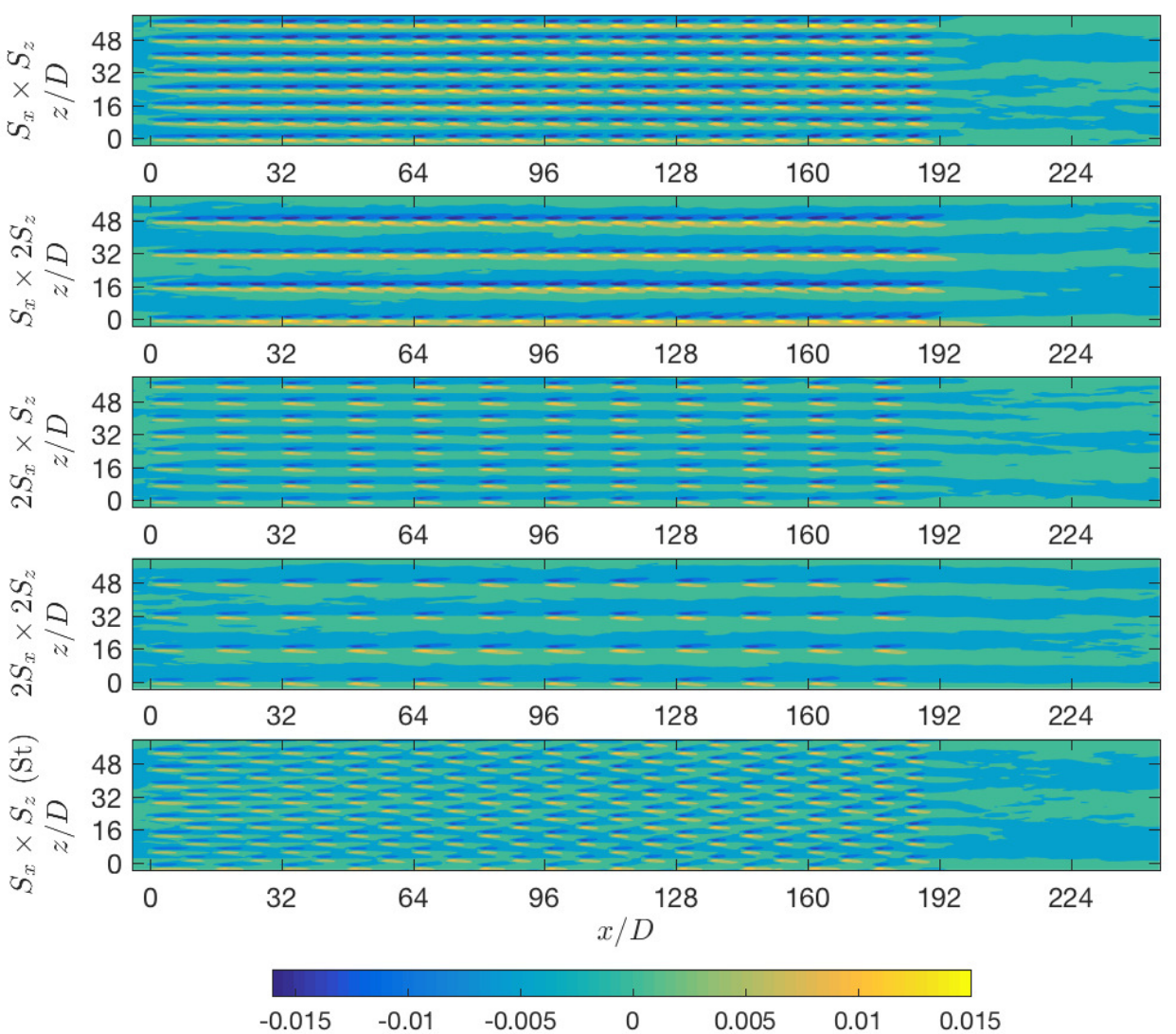

Figure 4.2: The normalized Reynolds shear stress, $\overline{u^{\prime} v^{\prime}} / U^{2}$, at hub-height for all 5 cases. Increasing the streamwise spacing, as seen in $2 S_{x} \times S_{z}$ and $2 S_{x} \times 2 S_{z}$, creates distinct breaks in Reynolds shear stress between each row implying that the Reynolds shear stress has less impact on subsequent turbines downstream for these cases.

though the staggered case has similar spacing to $S_{x} \times S_{z}$, the overall TKE levels are approximately $10 \%$ less than $S_{x} \times S_{z}$. 

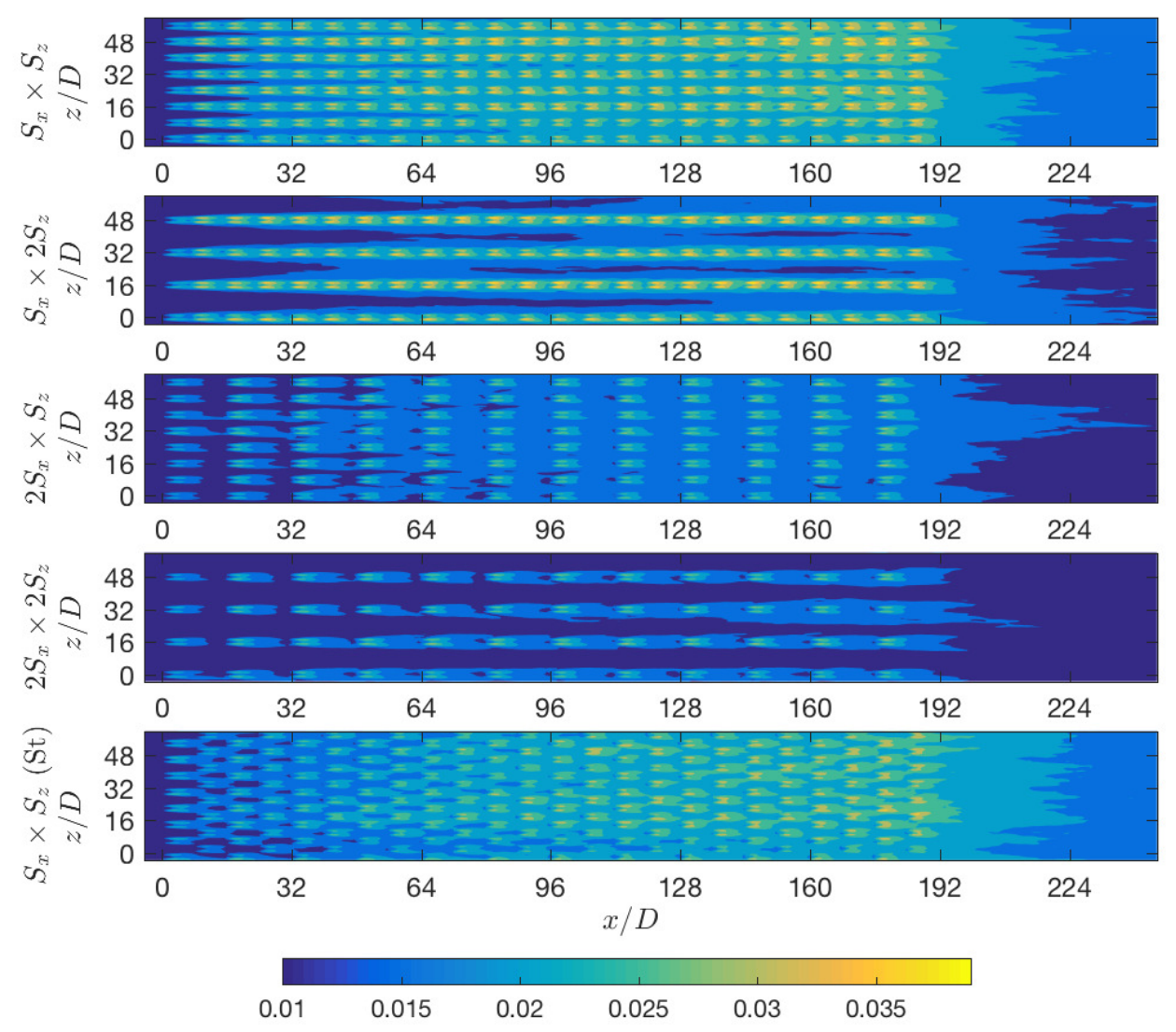

Figure 4.3: The normalized turbulence kinetic energy, $\frac{1}{2}\left(\overline{u^{\prime 2}}+\overline{v^{\prime 2}}+\overline{w^{\prime 2}}\right) / U^{2}$, at hub height for all 5 cases. TKE is highest further downstream in the domain and is particularly pronounced in the two cases with the least spacing in both directions, $S_{x} \times S_{z}$ and $S_{x} \times S_{z}(S t)$.

\subsubsection{Subdomains}

Analysis is broken up into the following symmetric subdomains: developing, fully developed and relaxed. Within the fully developed region, the far and near wake will be discussed as well. Figure 4.4A and table 4.1 outlines the developing, fully developed and relaxed subdomains in terms of streamwise distance, $x / D$. The subdomains are 


\begin{tabular}{cccccc}
\hline \hline Subdomain & $\mathrm{S}_{x} \times \mathrm{S}_{z}$ & $\mathrm{~S}_{x} \times 2 \mathrm{~S}_{z}$ & $2 \mathrm{~S}_{x} \times \mathrm{S}_{z}$ & $2 \mathrm{~S}_{x} \times 2 \mathrm{~S}_{z}$ & $\mathrm{~S}_{x} \times \mathrm{S}_{z}(S t)$ \\
\hline Developing & $0-24$ & $0-24$ & $0-32$ & $0-32$ & $0-24$ \\
Fully Developed & $144-168$ & $144-168$ & $128-160$ & $128-160$ & $144-168$ \\
Relaxed & $176-200$ & $176-200$ & $160-192$ & $160-192$ & $176-200$ \\
\hline
\end{tabular}

Table 4.1: Subdomains in terms of streamwise distance $(x / D)$ from the entry into the canopy for all cases.

symmetric and exclude the outer lateral turbines. Each subdomain begins directly upstream of a turbine row and ends either two or three rows downstream. The relaxed section for each case includes a streamwise distance past the last turbine row relative to each cases streamwise spacing (4D or $8 \mathrm{D})$. The developing region represents a part of the flow that is highly dependent on the streamwise location, $x$. The cases with the increased spacing in the streamwise, $2 S_{x} \times 2 S_{z}$ and $2 S_{x} \times S_{z}$, do not actually reach a fully developed flow as discussed in [28]. The fully developed subdomains for this study are chosen to represent the part of the flow where the flow is independent of the $x$ location. The relaxed region includes part of the domain past the last turbine to include flow recovery as the flow relaxes.

Figure $4.4 \mathrm{~B}$ outlines the symmetric near and far wake regions within the fully developed subdomain of each case. The near wake considers up to 3D past the turbine, whereas the far wake is $3 \mathrm{D}$ upstream from the preceding turbine. 

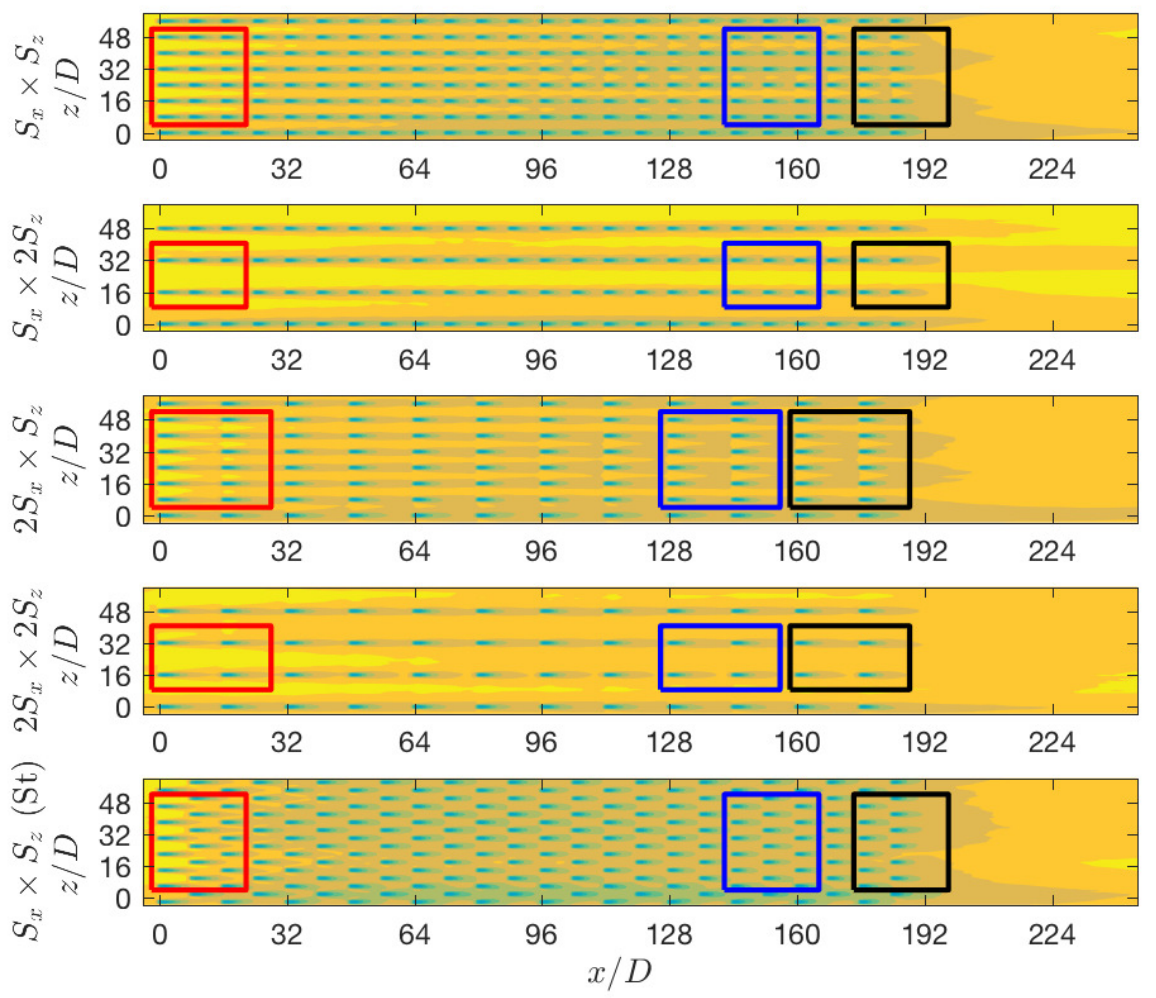

(A) Subdomains for Developing (red outline), Fully Developed (blue outline), and Relaxed (black outline) on top of the mean velocity of figure 4.1

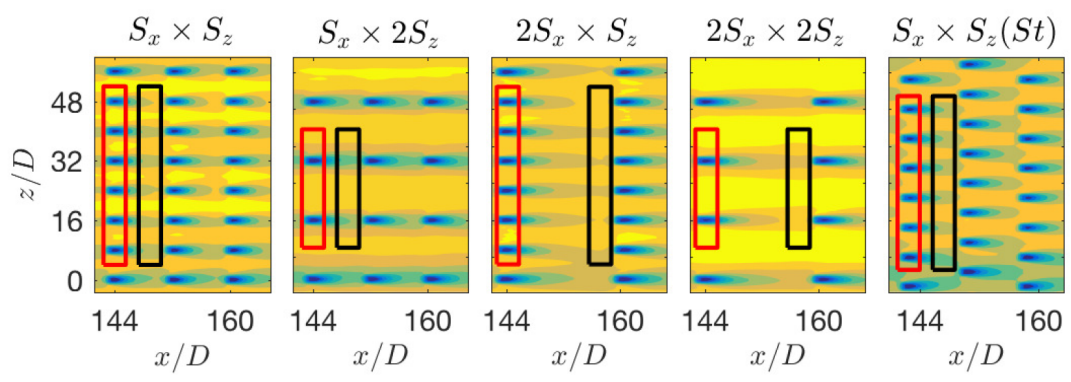

(B) Subdomains for Near Wake (red outline) and Far Wake (black outline)

Figure 4.4 


\section{Chapter 5}

\section{Results: Subdomain Statistics}

\subsubsection{Developing, Fully Developed and Relaxed}

\section{Reynolds Stress}

For a single wind turbine, the differences in upstream and downstream kinetic energy fluxes facilitate power extraction. On the other hand for large turbine arrays, turbulence is not produced but imported from above [6]. Kinetic energy is entrained from the flow above the turbines resulting in a vertical transport of energy in the boundary layer [6]. For a fully developed flow regime, variations in the vertical direction are found to be far more relevant in comparison to changes in the streamwise and spanwise directions. The vertical component is important for determining the efficiency of a turbine in large wind farms and is often represented with horizontally averaged variables [6]. Due to this vertical entrainment, most of the analysis hereon will be considering the vertical structure with flow variables horizontally averaged in the streamwise $(x)$ and spanwise $(z)$.

Vertical profiles of the normalized horizontally averaged Reynolds stresses, $\left\langle\overline{u^{\prime} u^{\prime}}\right\rangle / U^{2},\left\langle\overline{v^{\prime} v^{\prime}}\right\rangle / U^{2},-\left\langle\overline{u^{\prime} v^{\prime}}\right\rangle / U^{2}$, are plotted as functions of the normalized vertical height, $y / D$, as seen in Figure 5.1. These three Reynolds stresses will be discussed 
since they are highest in magnitude and most relevant due to its relationship with vertical transport. The Reynolds stresses determine which components are responsible for the production of TKE and the flux of kinetic energy. For all cases, $\left\langle\overline{u^{\prime} u^{\prime}}\right\rangle / U^{2}$ and $\frac{\partial}{\partial y}-\left\langle\overline{u^{\prime} v^{\prime}}\right\rangle / U^{2}$ are the highest contributor to the overall stress, and the most responsible for TKE production and kinetic energy flux. Figure 5.1A exhibits the dominance in $\left\langle\overline{u^{\prime} u^{\prime}}\right\rangle / U^{2}$ versus the other two Reynolds stresses, with values 3 times larger than $\left\langle\overline{v^{\prime} v^{\prime}}\right\rangle / U^{2}$ and order of magnitudes 3 times that of $-\left\langle\overline{\left\langle u^{\prime} v^{\prime}\right.}\right\rangle / U^{2}$. The normal streamwise Reynolds stress is most dominant due to the flow being forced in the streamwise $(u)$ direction while shearing with the surface, creating the ABL. The vertical derivative of $-\left\langle\overline{u^{\prime} v^{\prime}}\right\rangle / U^{2}$ is also high because energy is being entrained from above.

In the developing subdomain, $\left\langle\overline{u^{\prime} u^{\prime}}\right\rangle / U^{2}$ exhibits peak stress below the bottom rotor tip at $y / D=0.3$ for all cases except for the base and staggered case. The surface to bottom rotor tip between $y / D=0$ and $y / D=0.5$, is heavily affected by the ground surface roughness and near surface friction velocity thus showing increased magnitudes. Although for the more dense cases, $S_{x} \times S_{z}$ and $S_{x} \times S_{z}$ (St), peak magnitudes of the normal streamwise Reynolds stress is seen at the top rotor tip. This is caused by the increase in turbine obstruction with the flow decreasing the amount of shearing with the bottom surface, but increasing the amount of shearing at the top rotor tip. As the flow develops past the developing subdomain and the velocity deficit increases for all cases, shearing is diminished at the bottom surface. This results with a decrease in $\left\langle\overline{u^{\prime} u^{\prime}}\right\rangle / U^{2}$ at the bottom surface for all cases in the fully developed and relaxed subdomain. For all three subdomains, the staggered case has the lowest $\left\langle\overline{u^{\prime} u^{\prime}}\right\rangle / U^{2}$ until hub-height. Once at hub-height there is a sharp increase with the stress gradient, $\frac{\partial}{\partial y}\left\langle\overline{u^{\prime} u^{\prime}}\right\rangle / U^{2}$, until top rotor tip. This is also seen in the base case, $S_{x} \times S_{z}$. All cases have a steady decline 
in $\left\langle\overline{u^{\prime} u^{\prime}}\right\rangle / U^{2}$ once past the top rotor tip. Once in the fully developed subdomain, the variance between the cases increases from the hub to the top of the subdomain. Moving from fully developed to relaxed region, the slope of the Reynolds stress in all cases decreases between the bottom tip and top tip. The denser cases, $S_{x} \times S_{z}$ and $S_{x} \times S_{z}$ (St), have a smaller slope than the more sparse cases. $S_{x} \times S_{z}$ has a slope approximately $30 \%$ less than $2 S_{x} \times 2 S_{z}$.

In figure $5.1 \mathrm{~B}$, the developing region for $\left\langle\overline{\nu^{\prime} \nu^{\prime}}\right\rangle / U^{2}$ has a severe concavity from the ground up to its peak at the top rotor tip. Past the rotor tip, there is a sharp decline in the normal spanwise Reynolds stress which then eases once at $y / D=2.5$. The most dense cases, $S_{x} \times S_{z}$ and $S_{x} \times S_{z}$ (St), have the lowest Reynolds stress magnitude, whereas the most sparse case $2 S_{x} \times 2 S_{z}$ has the highest stress. The fully developed and relaxed regions exhibit similar trends such as the intensity of the concavity decreasing. $\frac{\partial}{\partial y}\left\langle\overline{v^{\prime} v^{\prime}}\right\rangle / U^{2}$ is small, emphasizing the minor role that $\left.\overline{\left\langle v^{\prime} v^{\prime}\right.}\right\rangle$ contributes to the overall flux and production of energy. The concavity in both these regions is still present, but less intensified as it gradually peaks at the rotor tip then steadily declines up past the given subdomain of $y / D=4$. Dissimilar to the developing region, in the fully developed and relaxed subdomains the most sparse case, $2 S_{x} \times 2 S_{z}$, displays the lowest magnitude in stress followed by the case with the increase in streamwise spacing, $2 S_{x} \times S_{z}$. The most dense cases, $S_{x} \times S_{z}$ and $S_{x} \times S_{z}$ (St), have the highest values of $\left\langle\overline{v^{\prime} v^{\prime}}\right\rangle / U^{2}$. All three subdomains for $\left\langle\overline{\left\langle v^{\prime} v^{\prime}\right.}\right\rangle / U^{2}$ show a sharp decrease in Reynolds stress from the ground to $y / D=0.15$, then an increase in stress occurs up until around the top rotor tip, where stresses in all cases begin to decline again. The peak magnitudes of $\left\langle\overline{v^{\prime} v^{\prime}}\right\rangle / U^{2}$ for the developing region is at $y / D=1.5$, but as the flow relaxes the peak stress propagates upward to $y / D=2$. 
The Reynolds shear stress in figure 5.1C has high fluctuations in the vertical transition resulting in a large vertical derivative of $-\left\langle\overline{u^{\prime} v^{\prime}}\right\rangle / U^{2}$. This vertical gradient is a crucial component of the mean energy equation demonstrating the significance of $-\left\langle\overline{u^{\prime} v^{\prime}}\right\rangle$. In the fully developed region, the vertical gradient is largest in the swept rotor area because the top rotor tip is extracting kinetic energy vertically from the upper ABL. $S_{x} \times S_{z}$ and $S_{x} \times S_{z}$ (St) have the largest magnitude Reynolds shear stress and vertical gradient with peaks at the top rotor tip. Past the top rotor tip, all cases begin to converge to zero. Once in the fully developed regime, the vertical gradient propagates upward due to the expansion of the turbine wake shifting the locations of the Reynolds shear stress. The high gradients now occur from $y / D=1.5$ to $y / D=2.5$. The variance between the cases increases as well, especially past the top rotor tip and $S_{x} \times 2 S_{z}$ has increased in magnitude five-fold. In the relaxed subdomain, the vertical gradient above the top tip has decreased. For $S_{x} \times 2 S_{z}$, the Reynolds shear stress magnitude has decreased and the vertical gradient has propagated even further between $y / D=2.5$ to $y / D=3.5$. This is in consequence to the high velocity channels that appear between the turbines pushing the gradient up.

\section{Dispersive Stress}

The vertical profiles of normalized dispersive stresses are outlined in figure 5.2. In the developing region for the normal dispersive streamwise stress, $\left\langle\overline{u^{\prime \prime} u^{\prime \prime}}\right\rangle / U^{2}$, shown in figure 5.2A, the denser canopies have a larger dispersive stresses than in the sparser canopies. The base case $S_{x} \times S_{z}$, is twice as large as the most sparse case, $2 S_{x} \times 2 S_{z}$. $\left\langle\overline{u^{\prime \prime} u^{\prime \prime}}\right\rangle / U^{2}$ takes on a parabolic shape, starting with negligible stresses at the surface, with a steep vertical gradient to a peak dispersive stress at hub-height $(y / D=1)$. Once 

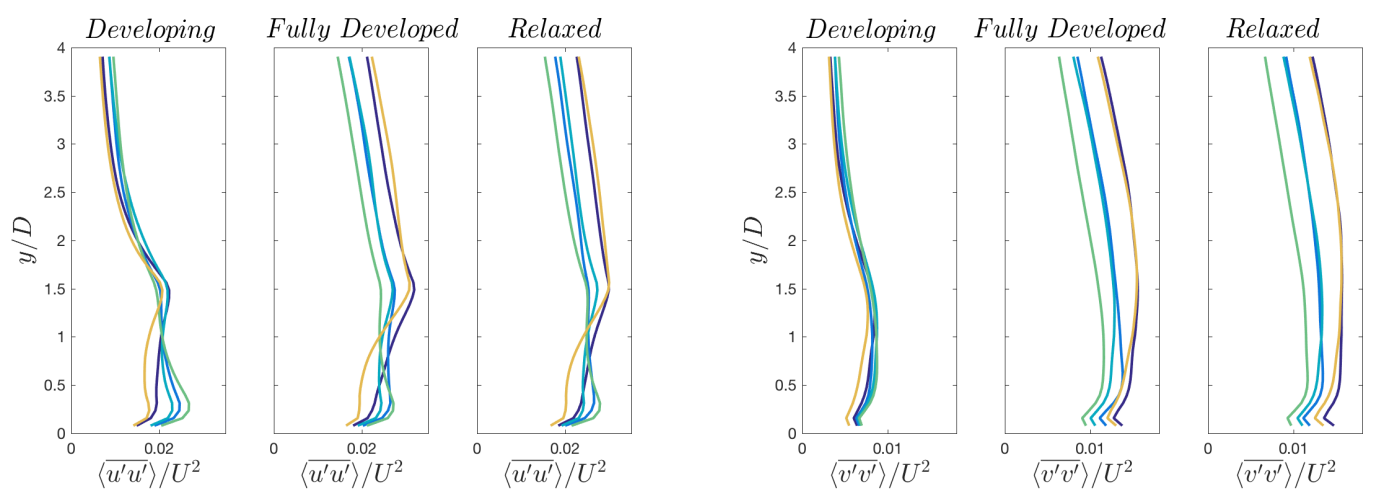

(A)

(B)

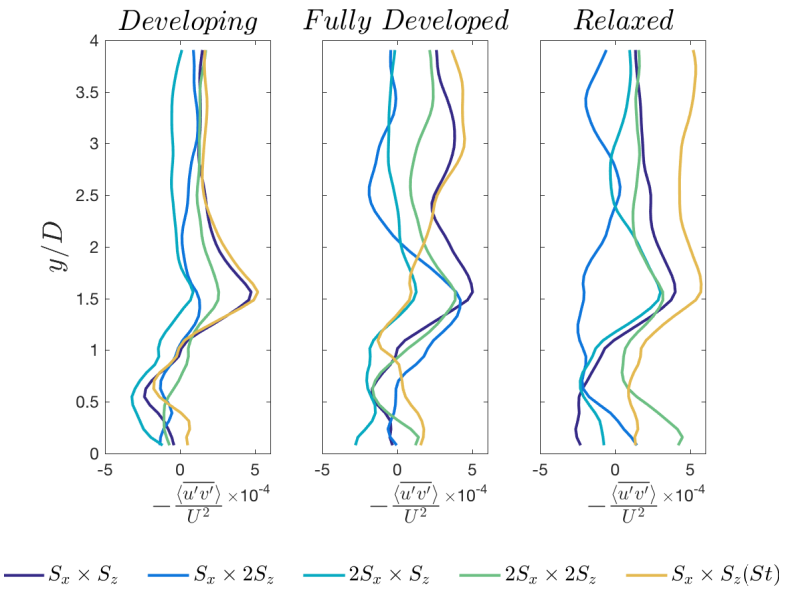

(C)

Figure 5.1: Normalized spatially averaged Reynolds stress in developing, fully developed and relaxed subdomain. $\left\langle\overline{u^{\prime} u^{\prime}}\right\rangle / U^{2}$ has the greatest magnitude among the three stresses.

at hub-height, the dispersive stress has another steep vertical gradient and declines till it is null right above the top tip at $y / D=2$. Large dispersive stresses are expected to center around the hub, since the hub and swept rotor area are the only contributors to the spatial variation in the flow.

In the fully developed region, there is a similar parabolic shape to the developing region, but $\left\langle\overline{\left\langle u^{\prime \prime} u^{\prime \prime}\right.}\right\rangle / U^{2}$ in the dense cases decreases by up to $50 \%$. This is a result from 
the large velocity deficit caused by the drag of the turbines. Denser canopies have more drag, diverting the flow around and above the canopy resulting in a decreased velocity and dispersive stress. As seen earlier in figure 4.1, the dense cases have the largest velocity deficit once past the entry region of the canopy. In the fully developed region, the staggered case now has the lowest dispersive stress, with the other cases (except for $S_{x} \times 2 S_{z}$ ) sharing similar magnitudes. The cases with an increase in spanwise spacing, $S_{x} \times 2 S_{z}$ and $2 S_{x} \times 2 S_{z}$, only decrease by $20 \%$. These two cases do not exhibit the same level of velocity deficit, as seen in the dense cases. An increase in spanwise spacing results in a diminished decay of normal streamwise dispersive stress causing $S_{x} \times 2 S_{z}$ to now have the largest dispersive stress in the fully developed region.

Once in the relaxed subdomain, the separation between dense and sparse cases increases as the two dense cases and $S_{x} \times 2 S_{z}$ decrease by approximately $10 \%$. The dense cases, $S_{x} \times S_{z}$ and $S_{x} \times S_{z}(S t)$ now have the lowest dispersive stress. Referring once again to figure 4.1, the more dense cases recover at a slower rate compared to the other cases past the last row of turbines. This causes a further decay in dispersive stress for the relaxed region.

Overall, the normal streamwise dispersive stress, $\left\langle\overline{u^{\prime \prime} u^{\prime \prime}}\right\rangle / U^{2}$, is approximately $60 \%$ less in fully developed and relaxed than in the developing region. These findings are similar to a study by Moltchanov [21], where dispersive stresses were larger at the entry region of the canopy. The high dispersive stresses in the developing region are a result of the relative high velocities in the entrance of the canopy. A large velocity difference caused by the onset of turbine between the high velocities at the entrance and the turbine wake regions and bottom surface results in a high dispersive stress. It is interesting to note that the dispersive stress range among the cases are not consistent in 
the three subdomains. In previous studies, dispersive stresses were found to be highest in sparse canopies [23], but this is only present in the fully developed and relaxed regions. This is because as the flow is developing the dispersive stresses in the dense cases decay at a faster rate than the sparse cases due to high velocity deficit that exists in the dense cases.

Figure 5.2B outlines $\left\langle\overline{v^{\prime \prime} v^{\prime \prime}}\right\rangle / U^{2}$ among the regions. The entire subdomain exhibits a similar parabolic shape as in figure 5.2A, but a sharp decrease at the hub-height, followed by a sharp increase till $y / D=1.1$, then a steady decline till the stress is null. The staggered case has the highest dispersive stress throughout the subdomain. Similar to figure 5.2A, the developed region has the overall highest stresses.

The shear stress component, $-\left\langle\overline{u^{\prime \prime} v^{\prime \prime}}\right\rangle / U^{2}$, shown in figure $5.2 \mathrm{C}$ displays a symmetric wave about the zero axis with a shift in the negative direction between the bottom rotor tip and hub. In the developing regime there is a noticeable variance among the cases with the denser cases showing the highest magnitude stress and the most sparse case, $2 S_{x} \times 2 S_{z}$, with the lowest magnitude stress. There is a high vertical derivative between the top and bottom rotor tip. Peak stresses are the areas between both tips and the hub. The stress is also nonexistent before the bottom tip and after the top tip. In the fully developed subdomain, trends are similar to the developing, but the overall stress has decreased by half for the two dense cases, $S_{x} \times S_{z}$ and $S_{x} \times S_{z}$ (St). The other cases decrease by only $10-15 \%$ causing there to be less variance among all cases. The case with the highest spacing, $2 S_{x} \times 2 S_{z}$, continues to exhibit the lowest magnitude stress. In the relaxed regime, there are similar symmetric trends shown in the other two subdomains. The two dense cases and $S_{x} \times 2 S_{z}$ continue to decrease at a faster rate then the sparse cases. This causes $2 S_{x} \times S_{z}$ to have the highest stress and vertical derivative 
in the relaxed region. For $-\left\langle\overline{u^{\prime \prime} v^{\prime \prime}}\right\rangle / U^{2}$, the streamwise dense cases decay at a faster rate than the cases with increased streamwise spacing.

Analogous to the Reynolds stress, the normal streamwise stress component, $\left\langle\overline{u^{\prime \prime} u^{\prime \prime}}\right\rangle / U^{2}$, dominates in comparison to $\left\langle\overline{v^{\prime \prime} v^{\prime \prime}}\right\rangle / U^{2}$ (2 order of magnitudes larger) and $-\left\langle\overline{u^{\prime \prime} v^{\prime \prime}}\right\rangle / U^{2}$ (1 order of magnitude larger). All three stresses decrease from downstream from the developing to the relaxed regions. The decreases in dispersive stress relates directly to the velocity deficit.

\section{Invariants}

For analyzing anisotropy within the canopy, the normalized anisotropic tensor, $b_{i j}$, is decomposed to obtain the eigenvalues as seen in equation 2.10. The two invariants, $\eta$ and $\xi$ will be derived from the normalized anisotropic tensor, $b_{i j} . \eta$ describes the local degree of three dimensionality in turbulence and $\xi$ describes the corresponding shape with F representing the combination of the two invariants. For this study, the invariants are spatially averaged in the streamwise $(x)$ and spanwise $(z)$ to study the vertical profile of the invariants in the flow. Figure 5.3 shows the spatially averaged invariants, $\langle\xi\rangle,\langle\eta\rangle$ and $\langle F\rangle$ for developing, fully developed, and relaxed subdomains.

The invariant $\langle\eta\rangle$ shows the degree of anisotropy and is depicted in figure 5.3A. Referring to the developing flow, there is a small amount of variation among the cases. All cases are consistently within $10 \%$ of each other with the maxima $\langle\eta\rangle=0.225$. The bottom surface before the bottom rotor tip is the most anisotropic with a sharp increase from the surface to the maxima at $y / D=0.2$. High anisotropy at the bottom surface is expected due to the shearing of the wind against the surface. Once at the bottom rotor 


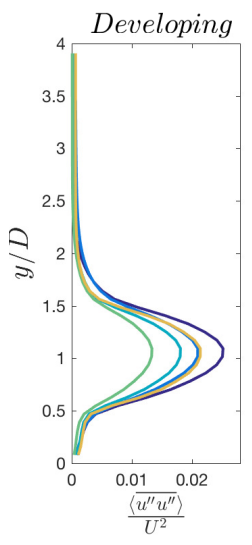

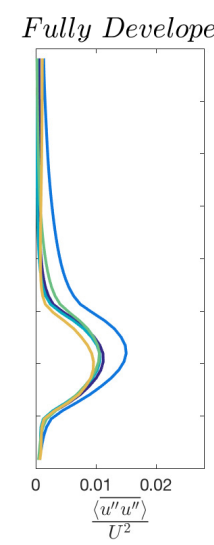

(A)
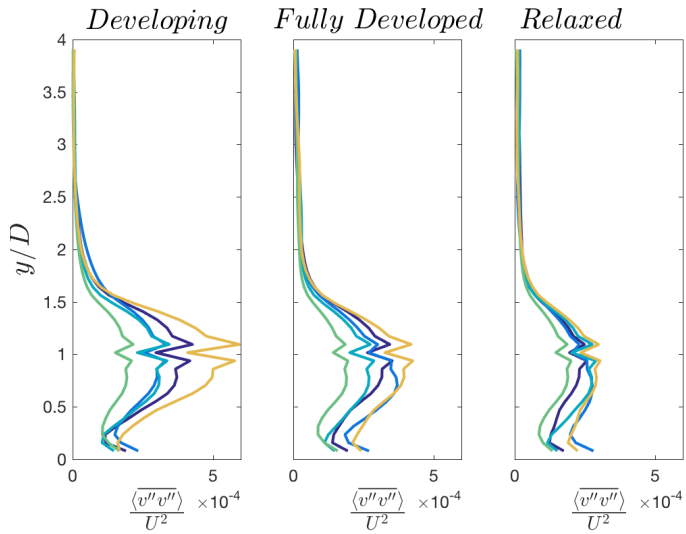

(B)
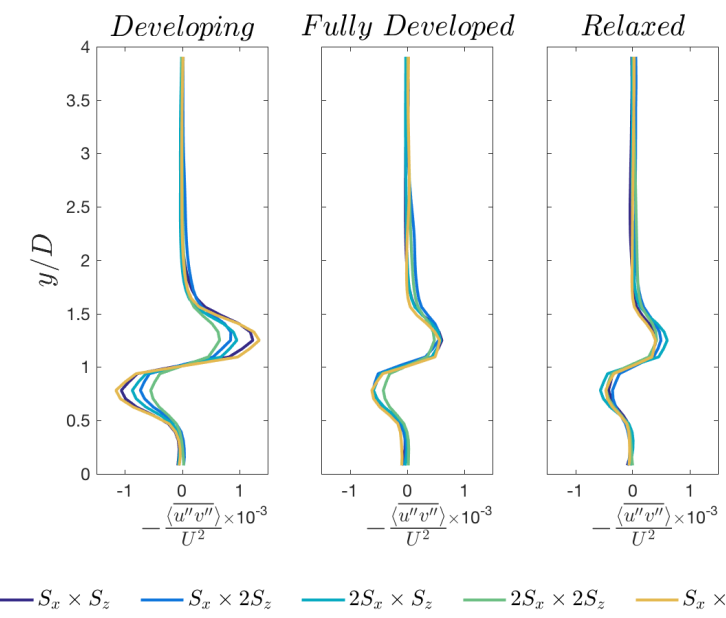

$-S_{x} \times S_{1}$

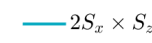

$-2 S_{x} \times 2 S_{z} \quad-S_{x} \times S_{z}(S t)$

(C)

Figure 5.2: Spatially averaged dispersive stress in developing, fully developed and relaxed subdomain. Stresses follow a parabolic trend with the peak stress at hubheight. Developing subdomain has the highest stress, with the relaxed region with the lowest stress magnitude. 
tip, there is a decline in $\eta$ up to the hub, especially for the staggered case. The hub is a blunt body which disorganizes the flow as it moves downstream. This disorganization causes an increase an isotropy in the flow which results in a sharp decline in $\eta$ at the hub. From hub-height to the top tip, there is an increase in anisotropy due to the shearing with the top rotor tip. The staggered case and base case show the highest increase in $\langle\eta\rangle$ at the top rotor tip emblematic to more anisotropy caused by the presence of more turbine rotors to shear with. In all five cases, $\langle\eta\rangle$ converges at the top tip displaying a return to isotropy which occurs with the decay of turbulence.

Once in the fully developed flow, there are overall higher levels of isotropy and greater variation among the cases (up to $20 \%$ ), particularly at $y / D=0.6$. The staggered case is the most isotropic with the highest degree of isotropy between the bottom rotor tip and hub-height. All cases exhibit a sharp increase in anisotropy at the top rotor tip, but not as pronounced as seen in the developing region. The cases with an increase in spanwise spacing $2 S_{x} \times 2 S_{z}$ and $S_{x} \times 2 S_{z}$ are the most anisotropic.

As the flow relaxes, all cases remain similar to the fully developed region except for $2 S_{x} \times 2 S_{z}$ which sees a $10 \%$ increase in anisotropy. This is because the relaxed regime for the sparse case has the last amount of turbines to obstruct the shearing that occurs at the bottom surface. There are also less turbines for isotropy in the hub area as well. This sparse cases also sees a less rapid approach to isotropy past the top rotor tip due to the high amount of anisotropy below the top tip. Once again, the staggered case displays the highest amount of isotropic behavior, followed by the base case of $S_{x} \times S_{z}$.

Positive values of $\xi$ attribute to a stretched spheroid and negative values mean the shape is squeezed, or oblate. The values $\langle\xi\rangle$, shown in figure 5.3B for developing regions contains only positive values, this is indicative to only one-component turbu- 
lence with a prolate spheroid shape. On the other hand, fully developed and relaxed regions contain negative $\xi$ values from the surface to the hub-height, especially for the denser cases, $S_{x} \times S_{z}$ and $S_{x} \times S_{z}$ (St). These negative values indicate oblate spheroid shapes.

Figure 5.3C combines the two invariants, $\eta$ and $\xi$ to create a function, $F$ that describes anisotropy as a range from 0 to $1 . F=0$ measures the approach to a twocomponent, anisotropic turbulence, whereas $F=1$ is a the approach to isotropic turbulence. In the developing regime all five cases have minimal variability. All cases have high anisotropy at the surface, as they transition vertically the cases approach isotropy with a peak in anisotropic behavior at the top tip. In the fully developed regime, the staggered case has a sharp shift in isotropy from the bottom tip to hub-height then converges to a stable value of $F$ that remains consistent to the top of the subdomain. In the relaxed subdomain, the most sparse case, $2 S_{x} \times 2 S_{z}$, return to isotropy at a slower pace than the other cases. This case followed by $S_{x} \times 2 S_{z}$ are the most anisotropic.

These correlations demonstrate that more sparse canopies are related to higher anisotropy. In the fully developed and relaxed regions, cases with larger spacing between turbines in the spanwise, $2 S_{x} \times S_{z}$ and $S_{x} \times 2 S_{z}$, reveal higher amounts of anisotropy below the top tip. At the top tip, an increase in streamwise spacing, $2 S_{x} \times S_{z}$ and $2 S_{x} \times S_{z}$, reveals higher anisotropy. As the flow moves downstream, the hub acts as blunt body that causes disorganization as it collides with the flow. This disorganization is isotropic. When there is more space between the turbines, it allows for the velocity deficit caused by a blunt body in the flow to recover and become more organized. The more organized a flow is, the more anisotropic it is. Because of this, more spacing between turbines results in higher anisotropy in the flow. 


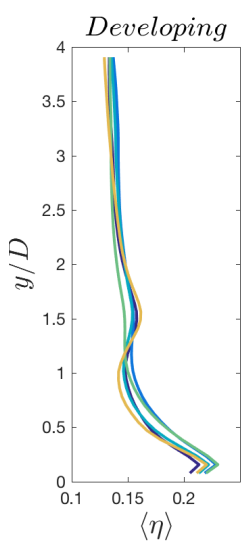

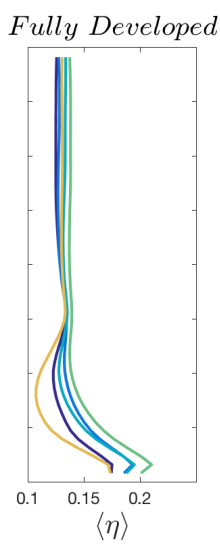

(A)
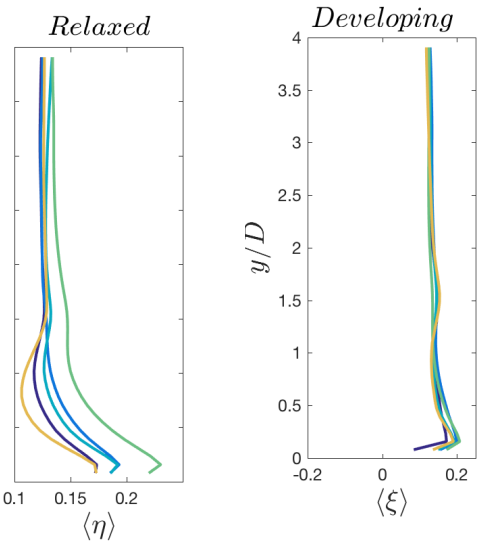

)

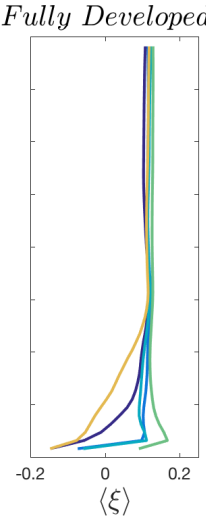

(B)
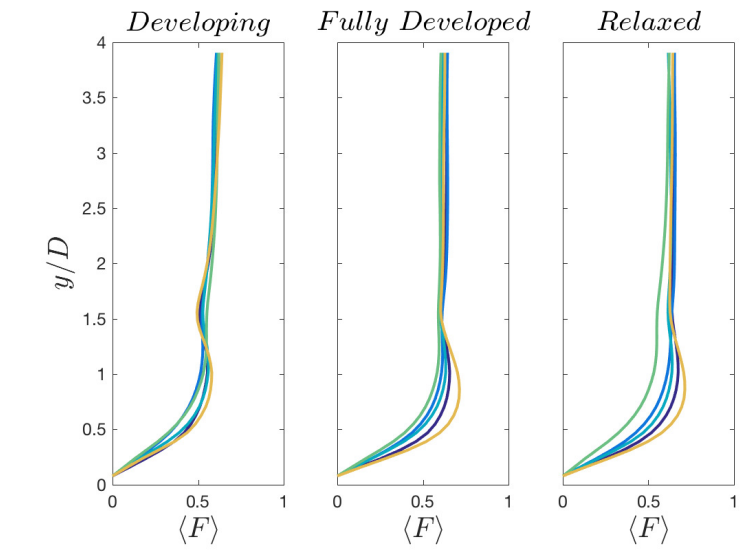

$-S_{x} \times S_{z} \quad-S_{x} \times 2 S_{z}$

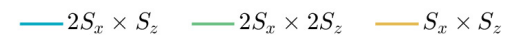

(C)

Figure 5.3: Spatially averaged invariants in the developing, fully developed and relaxed subdomains. Cases with the least amount of spacing between turbines, $S_{x} \times S_{z}$ and $S_{x} \times S_{z}$ (St), exhibit the most anisotropic behavior. 


\section{Chapter 6}

\section{Wake Analysis}

\subsubsection{Reynolds Stress}

Figure 6.1 shows the horizontally averaged Reynolds stresses for the near and far wake locations within the fully developed region. Similar to figure $5.1,\left\langle\overline{u^{\prime} u^{\prime}}\right\rangle / U^{2}$ has the highest stress in comparison to $\left\langle\overline{v^{\prime} v^{\prime}}\right\rangle / U^{2}$ and $-\left\langle\overline{u^{\prime} v^{\prime}}\right\rangle / U^{2}$. In the near wake for the normal streamwise Reynolds stress seen in figure 6.1A, the Reynolds stress peaks at the top tip then sharply declines past the top tip. Below the top tip, the three spaced cases are dominant. Then once at and above the top tip the dense cases, $S_{x} \times S_{z}$ and $S_{x} \times S_{z}$ (St), have the highest $\left\langle\overline{\left\langle u^{\prime} u^{\prime}\right.}\right\rangle / U^{2}$.

As the flow moves from the near wake to the far wake there is an overall $\left\langle\overline{u^{\prime} u^{\prime}}\right\rangle / U^{2}$ decay of approximately 13\%. The differences in magnitudes between the cases remain consistent at $25 \%$. The most dense cases show the highest Reynolds stress at $y / D=0.3$. In the far wake, the flow is less influenced by the turbines, which cause an increase in Reynolds stress near the bottom surface and a decrease in Reynolds stress at the top tip. This is especially noticeable in the cases with an increase in the streamwise spacing, $2 S_{x} \times S_{z}$ and $2 S_{x} \times 2 S_{z}$, since the far wake for those cases are further downstream from the turbines. Increasing the spanwise distance, $S_{x} \times 2 S_{z}$ has a similar trend but not nearly as pronounced, meaning that spanwise spacing does have as much of an effect 
on the wake.

In figure $6.1 \mathrm{~B}$, for $\left\langle\overline{\left\langle v^{\prime} v^{\prime}\right.}\right\rangle / U^{2}$, there are insignificant differences in the near and far wake with similar trends to the fully developed and relaxed regions of figure 5.1B. This is suggestive to the wake not having a large impact on the normal spanwise Reynolds stress. There is a concavity present that gradually peaks at the rotor tip then steadily declines up past the given domain of $y / D=4$. The dense cases, $S_{x} \times S_{z}$ and $S_{x} \times S_{z}$ (St), depict the highest values of $\left\langle\overline{\left\langle v^{\prime} v^{\prime}\right.}\right\rangle / U^{2}$, and the most sparse case, $2 S_{x} \times S_{z}$, has the lowest values.

The Reynolds shear stress, $-\left\langle\overline{u^{\prime} v^{\prime}}\right\rangle / U^{2}$, in figure $6.1 \mathrm{C}$ once again shows high vertical gradients in both the near and far wake. In the near wake, the vertical gradient of the Reynolds shear stress is largest within the swept rotor area, caused by the extraction of energy vertically from above. In the far wake, the vertical gradient decreases and is now not confined to the swept rotor area, but has propagated vertically. This is suggesting that the near wake dictates the extraction of energy in the flow. In the far wake, the variability among the cases increases as the effect from the turbines on the wake decreases. The cases with an increase in streamwise spacing exhibit a large decline in the Reynolds shear stress vertical gradient, especially for the case $2 S_{x} \times S_{z}$.

\subsubsection{Dispersive Stress}

The dispersive stresses in the near and far wake are shown in figure 6.2. It is clear that the dispersive stresses are not prevalent in the far wake. Looking at the near wake in figure 6.2A, the normal streamwise dispersive stress has the parabolic shape between the lower and top rotor tip. There is high variance between the cases with a difference of $50 \%$ between the lowest case, $S_{x} \times S_{z}$ (St), and the highest case, $2 S_{x} \times S_{z}$. A sparser 

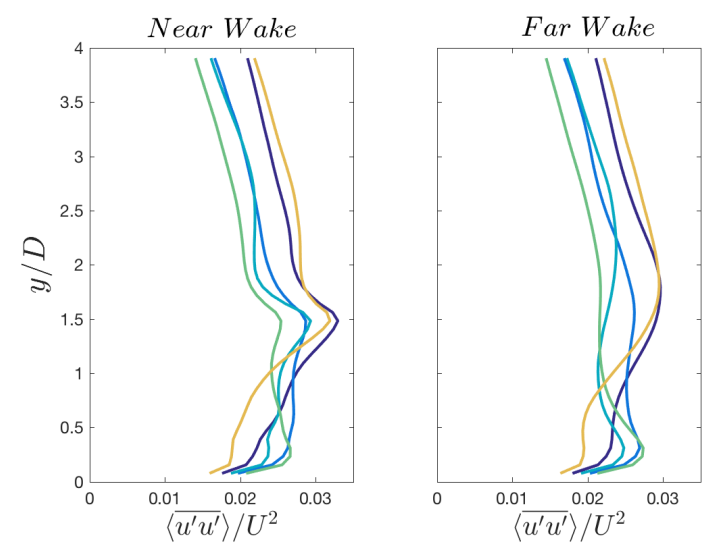

(A)

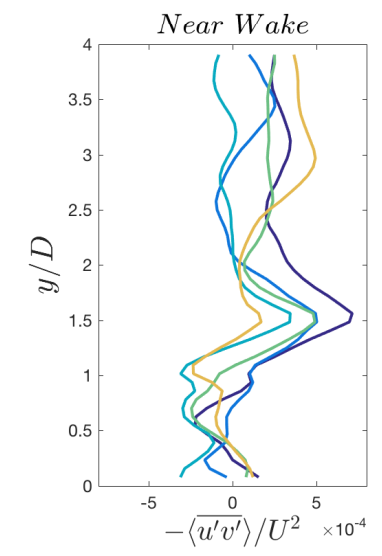

$-S_{x} \times S_{z} \quad-S_{x} \times 2 S_{z} \quad-2 S_{x} \times S_{z}$
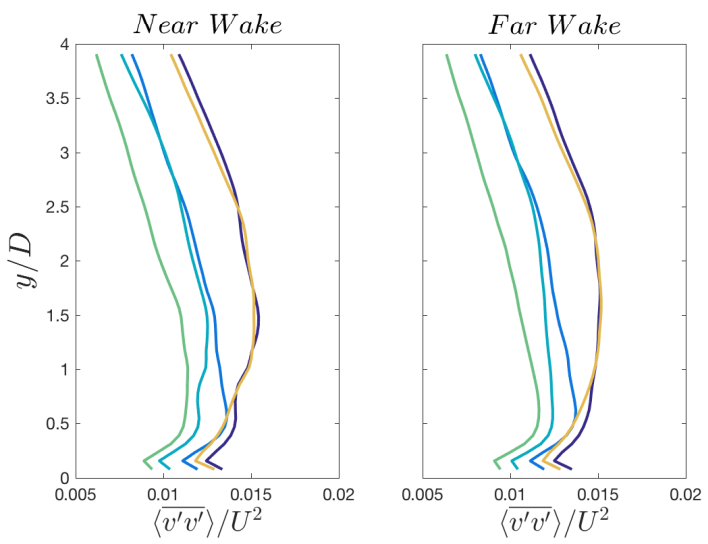

(B)

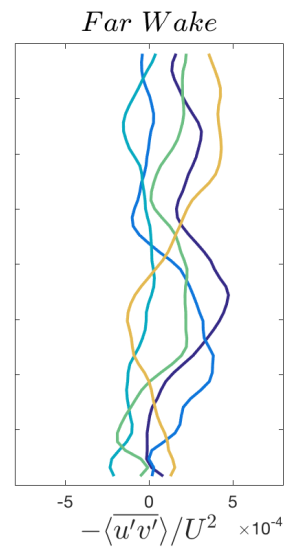

(C)

Figure 6.1: Spatially averaged Reynolds stress in the near and far wake. The near wake contains the highest contribution from the stresses.

canopy in the streamwise direction correlates to a higher dispersive stress in the near wake. The most dense cases $S_{x} \times S_{z}(\mathrm{St})$ and $S_{x} \times S_{z}$ have the lowest overall $\left\langle\overline{u^{\prime \prime} u^{\prime \prime}}\right\rangle / U^{2}$. In the far wake, the dispersive stress has decreased by over an order of magnitude and is negligible for all cases except $S_{x} \times 2 S_{z}$. The parabolic is present but has diminished greatly.

In figure 6.2B, the near wake of the normal spanwise dispersive stress resembles the 
parabolic shape seen earlier in the subdomains of figure 5.2B. There is a sharp decrease at the hub-height, followed by a sharp increase till $y / D=1.1$, then a steady decline till the stress is null. There is no transparent relationship between spacing and magnitude of $\left\langle\overline{v^{\prime \prime} v^{\prime \prime}}\right\rangle / U^{2}$. Case $2 S_{x} \times S_{z}$ has the highest dispersive stress, while $S_{x} \times S_{z}$ has the lowest dispersive stress. In the far wake, the profiles lose their shape completely and decrease by over an order of magnitude, highlighting the insignificance of the normal spanwise dispersive stresses in the far wake.

The near wake for the dispersive shear stress in figure $6.2 \mathrm{C}$ also has similar trends to the subdomains of figure $5.2 \mathrm{C}$. There is a high vertical derivative in the swept rotor area. The dispersive shear stress is approximately zero at the hub-height and maximum in the areas between both tips and hub. $\left\langle\overline{u^{\prime \prime} v^{\prime \prime}}\right\rangle / U^{2}$ is nonexistent before the bottom tip and after the top tip. Increased spacing in the streamwise correlates to a higher dispersive shear stress and vertical derivative. Case $2 S_{x} \times S_{z}$ has the highest magnitudes, followed by case $2 S_{x} \times 2 S_{z}$. Increasing the spanwise spacing results with the lowest dispersive shear stress and vertical derivative between the cases. The dispersive shear stress decreases dramatically and becomes negligible in the far wake.

These three dispersive stresses in figure 6.2 demonstrate that the momentum induced by spatial variations is highly influenced by the near wake. Once in the far wake the flow is not affected by the dispersive stress. Increasing the streamwise spacing correlates to higher dispersive stress, but increasing the spanwise spacing results with a decrease in dispersive stress. The dense cases have the lowest shear and normal streamwise dispersive stress. 


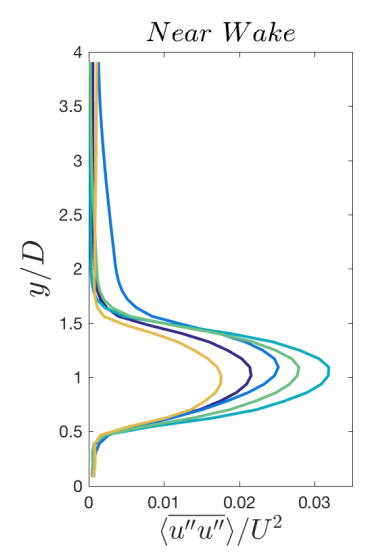

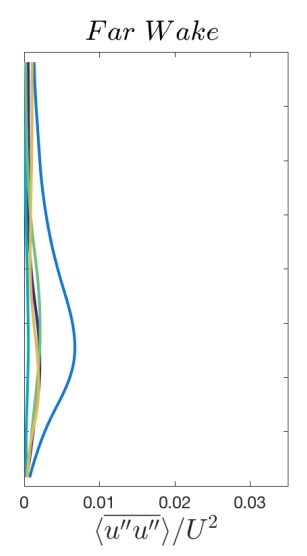

(A)

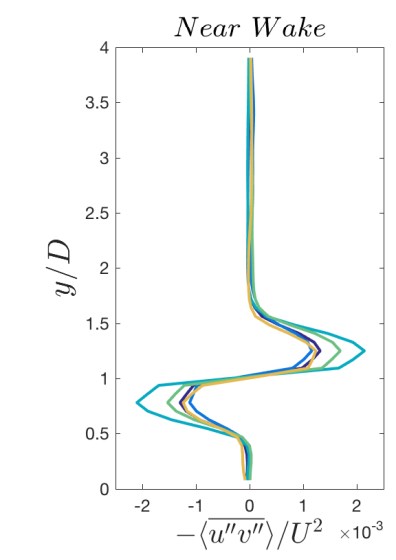

$-S_{x} \times S_{z} \quad-S_{x} \times 2 S_{z} \quad-2 S_{x} \times S$

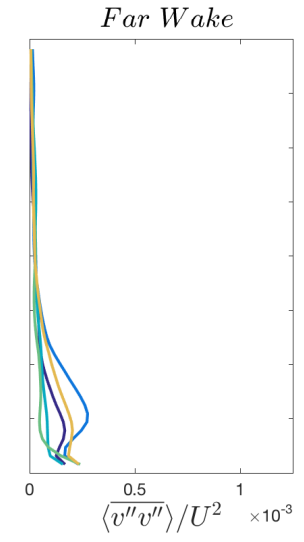

(B)

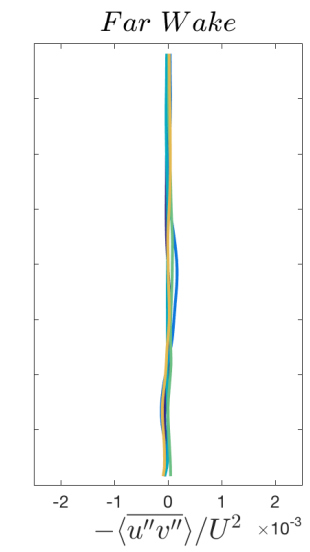

C)

Figure 6.2: Spatially averaged dispersive stress in the near and far wake. The momentum induced by spatial variations is highly influenced by the near wake.

\subsubsection{Invariants}

Unlike the dispersive stresses, the invariants in the near and far wake do not have stark differences. In the near wake of figure 6.3A, there are peaks of anisotropy near the surface at $y / D=0.2$ and at the top rotor tip. In between these two points, there is an increase in isotropy particularly between the bottom rotor tip and hub. Cases exhibit 
up to $20 \%$ of variability amid each other. The staggered case has the most isotropic behavior, but slightly above the top tip, the staggered case becomes more anisotropic than $S_{x} \times 2 S_{z}$ and $S_{x} \times S_{z}$. Increasing the spacing in both direction results in more anisotropic behavior. The most sparse case, $2 S_{x} \times 2 S_{z}$ is the most anisotropic. In the far wake, variability among the cases decreases to $13 \%$. The peak in anisotropy at the top rotor tip decreases. The two dense cases, $S_{x} \times S_{z}$ and $S_{x} \times S_{z}$ (St) are still the most isotropic.

The values of $\langle\xi\rangle$, shown in figure 6.3B contain both positive and negative values in the near and far wake. The only case that does not have any negative values is $2 S_{x} \times 2 S_{z}$, evident in both wakes. This means that increasing the spacing in both directions results in one-component turbulence with a prolate spheroid shape. Both near and far wake show similar trends. Negative values occur from the surface to the hub-height, especially for the denser cases, $S_{x} \times S_{z}$ and $S_{x} \times S_{z}$ (St). A dense canopy proves to have more oblate shapes of turbulence. Cases $S_{x} \times 2 S_{z}$ and $2 S_{x} \times 2 S_{z}$ have the least amount of variability between hub-height and $y / D=2$ for both near and far wake, which could entail that an increase in spacing in the spanwise is correlated to a decrease in variability of anisotropic shapes from hub height to above the top tip.

When measuring the approach to either isotropic or anisotropic turbulence in the near wake shown in figure 6.3C, all cases move toward isotropy from the surface up until the hub. Once at the hub, there is a sharp increase in anisotropy to the top rotor tip. The staggered case has the largest shift towards isotropy from the bottom tip to hub height then converges to a stable value of $F$ that remains consistent to the top of the domain. In the far wake, the anisotropic behavior at the top rotor tip is less pronounced. In both wake locations, the staggered case is the most isotropic, whereas 
the most spaced $2 S_{x} \times 2 S_{z}$ is the most anisotropic. 


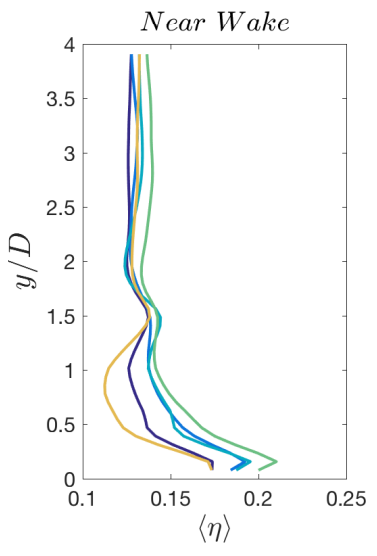

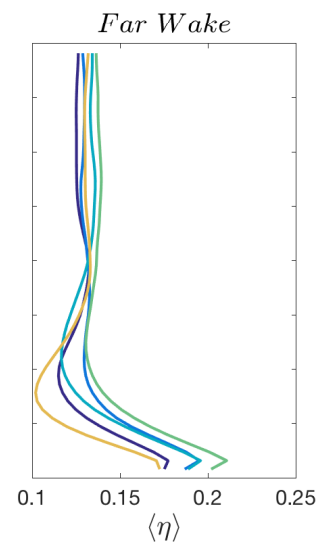

(A)

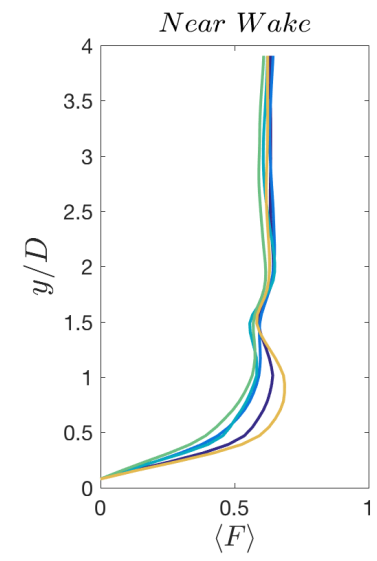

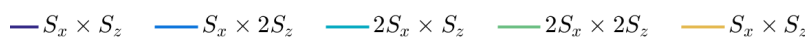

(C)
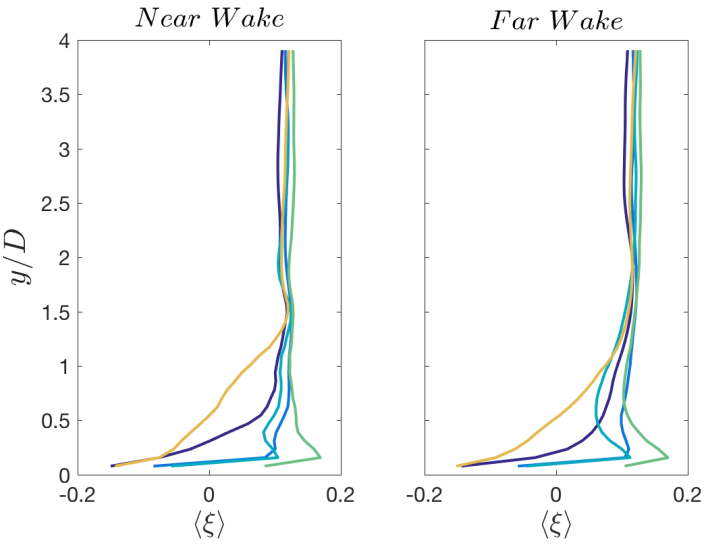

(B)

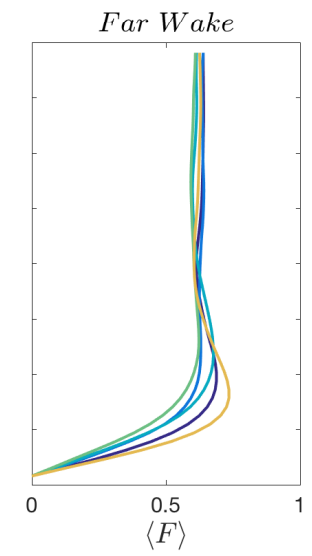

Figure 6.3: Spatially averaged invariants in the near and far wake. In both wake locations, the staggered case is the most isotropic, whereas the most spaced $2 S_{x} \times 2 S_{z}$ is the most anisotropic. In the near wake, there is a spike in anisotropic turbulence at the top rotor tip. This spike decays significantly in the far wake. 


\section{Chapter 7}

\section{Scatterplots}

Figure 7.1, 7.2, 7.3, 7.4 are scatter plots relating either Reynolds or dispersive stresses to the three invariants for the near wake in the fully developed region. Only the two most extreme cases, $S_{x} \times S_{z}$ and $2 S_{x} \times 2 S_{z}$ as well as the staggered case, $S_{x} \times S_{z}$ (St) will be compared to highlight the differences between a dense vs. sparse canopy. The plots are color coded according to vertical height $y / D$. The vertical volume is truncated to $y / D=2$ for the Reynolds and dispersive stress plots given that activity for the dispersive and Reynolds stress is restricted to the canopy region. For the dispersive stresses, the vertical volume from $0.5 \leq y / D \leq 1.5$ is color coded while the areas below the bottom rotor tip $(y / D=0.5)$ and above the rotor tip $(1.5<y / D \leq 2)$ are colored in black. That is done due to the dispersive stresses are only prevalent in the swept rotor area. Quantities are not spatially averaged since it is of interest to capture the spatial variations as well.

\subsubsection{Reynolds Stress: $S_{x} \times S_{z}$}

Figure 7.1 is a scatter plot for the Reynolds stresses of the base case, $S_{x} \times S_{z}$. The plots in figure 7.1 compare the three normalized Reynolds stresses, $\overline{u^{\prime} u^{\prime}} / U^{2}, \overline{v^{\prime} v^{\prime}} / U^{2}$, and $-\overline{u^{\prime} v^{\prime}} / U^{2}$ with the two invariants $\eta, \xi$, and the function $F$. When considering the 
Reynolds stress $\overline{u^{\prime} u^{\prime}} / U^{2}$ vs. $\eta$, most of the values of $\eta$ lie between 0.05 and 0.225 . There is a linear correlation between the magnitude of Reynolds stress and $\eta$. As the normal streamwise Reynolds stress increases, so does $\eta$. The highest values of $\overline{u^{\prime} u^{\prime}} / U^{2}$ and anisotropy occur slightly below and above the rotor tip from $y / D=1.3-2$. The area between the surface and bottom rotor tip also experiences high anisotropy, but with a low $\overline{u^{\prime} u^{\prime}} / U^{2}$ magnitude. When comparing $\overline{u^{\prime} u^{\prime}} / U^{2}$ and $\xi$, all of the negative values occur between the surface and bottom rotor tip where the stress is actually at its lowest. There are a greater number of points with positive $\xi$ values meaning that more prolate spheroid shapes in the turbulence, particularly at and above the bottom tip. A linear relationship is also present with an increase in normal streamwise Reynolds stress relating to an increase in $\xi$. The highest point of $\overline{u^{\prime} u^{\prime}} / U^{2}$, is from the hub to right above the top tip where $\xi$ is positive and at its highest values. For $\overline{u^{\prime} u^{\prime}} / U^{2}$ and $F$, the linear correlation is also present. The bottom surface and top tip is the most anisotropic. At some peak stress levels where $\overline{u^{\prime} u^{\prime}} / U^{2}=3$, the flow is more anisotropic than at lower stress levels with the exception of the bottom surface. The flow is most isotropic around the hub, $y / D=1$, and above the rotor area at $y / D=2.5$.

The relationship between the stress and invariants is less obvious for the normal spanwise Reynolds stress. This is due to $\overline{v^{\prime} v^{\prime}} / U^{2}$ having the smallest impact on flux and production in comparison to the other two Reynolds stresses. For $\eta$, the highest degree of anisotropy is around the top tip ( $y / D=1.3-1.8)$, where the greatest magnitude of $\overline{v^{\prime} v^{\prime}} / U^{2}$ is also present. For the invariant, $\xi$, negative values exist close to the bottom surface, where values of $\overline{v^{\prime} v^{\prime}} / U^{2}$, are comparable to higher vertical locations near the hub. $F$ shows how the surface is the most anisotropic with the most anisotropic behavior occurring right above the top tip and the hub with the most isotropic turbulence. 
The Reynolds shear stress, $-\overline{u^{\prime} v^{\prime}} / U^{2}$, is symmetric along the zero stress axis for all the invariants. Similar to $\overline{u^{\prime} u^{\prime}} / U^{2}$, there is a linear relationship between Reynolds shear stress and $\eta$. As the Reynolds shear stress magnitude increases, so does $\eta$ and anisotropy in the turbulence. $\eta$ is highest at the bottom surface and the top tip. For the invariant, $\xi$, negative values are close to the bottom surface. $F$ is also symmetric with a linear relationship between the magnitude of $-\overline{u^{\prime} v^{\prime}} / U^{2}$, and anisotropy with the exception of the bottom surface.
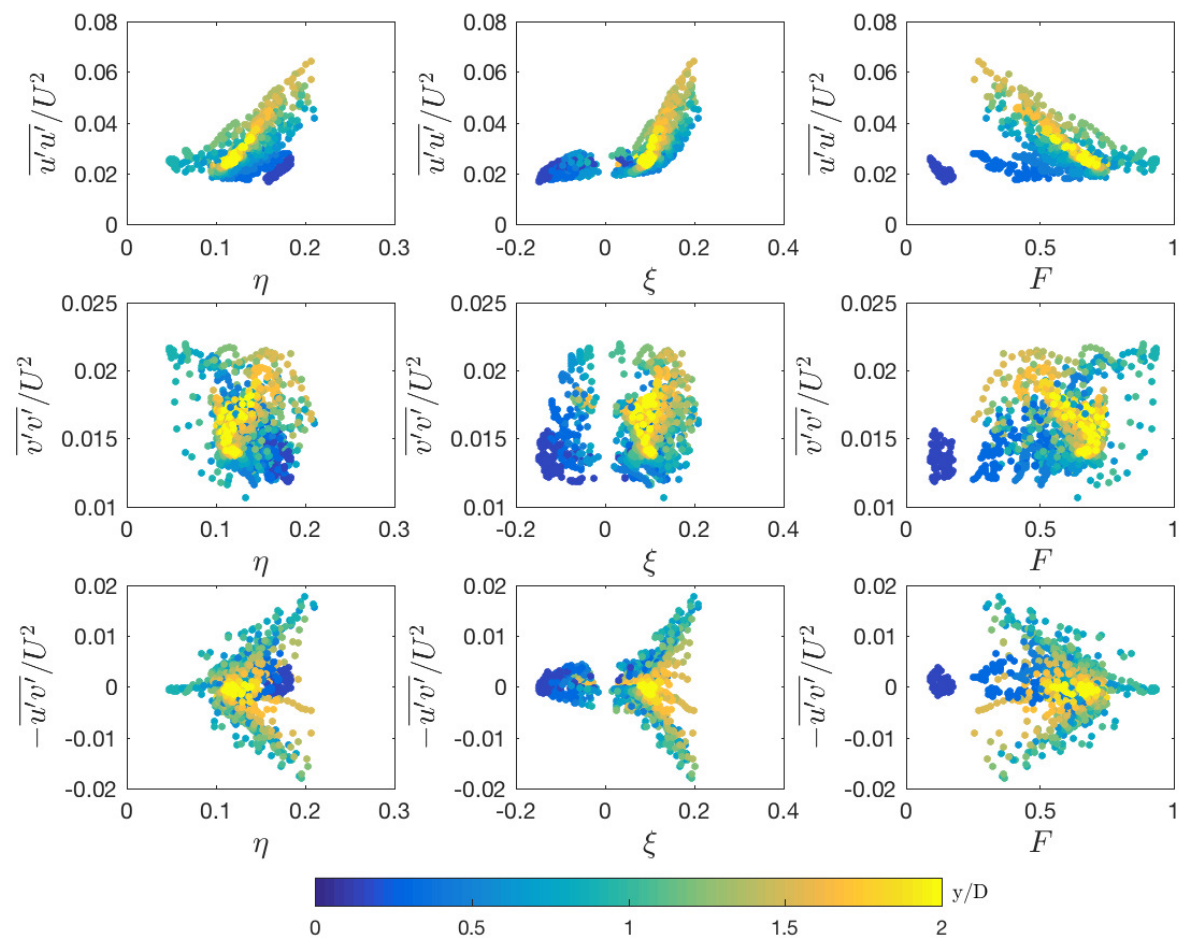

Figure 7.1: Reynolds stresses in the near wake in the fully developed regime for case $S_{x} \times S_{z}$. There is a linear correlation between the magnitude of Reynolds normal streamwise and shear stress and anisotropy. 


\subsubsection{Dispersive Stress}

Figure 7.2 compares the normalized dispersive stress, $\overline{u^{\prime \prime} u^{\prime \prime}} / U^{2}$, with the two invariants $\eta$, $\xi$, and $F$ for cases $S_{x} \times S_{z}, 2 S_{x} \times 2 S_{z}$ and $S_{x} \times S_{z}(\mathrm{St})$. For the base case of $S_{x} \times S_{z}$ most of the points for $\overline{u^{\prime \prime} u^{\prime \prime}} / U^{2}$ have dispersive stresses less than 0.05 , but some points between the hub and top rotor tip have high dispersive stresses with magnitudes twice that of the corresponding normal Reynolds stress. The normal streamwise dispersive stress increases when the spacing in the spanwise and streamwise are doubled with the maxima increasing from $\overline{u^{\prime \prime} u^{\prime \prime}} / U^{2}=0.16$ in the base case to $\overline{u^{\prime \prime} u^{\prime \prime}} / U^{2}=0.25$. However, the staggered case has a decrease in $\overline{u^{\prime \prime} u^{\prime \prime}} / U^{2}$ from the base case by approximately $15 \%$. In all three cases, below the bottom rotor tip and above the top rotor tip are areas of negligible dispersive stress, colored in black.

The plot of $\overline{u^{\prime \prime} u^{\prime \prime}} / U^{2}$ vs. $\eta$ for all three cases has a parabolic shape, with the highest stresses lying midway between the most and least anisotropic behavior, but leaning more toward isotropy. In the three cases, $\eta$ is largest at the top rotor tip with the most isotropic behavior at the hub. $\xi$ also has a similar parabolic trend that $\eta$ revealed. The negative $\xi$ values of the three cases are between the bottom rotor tip and hub, but by increasing the spacing, there is an increase in positive values for $\xi$, indicating more prolate spheroid shapes. Increased spacing also resulted in a shift toward more anisotropy. $\eta$ now ranges from 0.06 to 0.23 compared to the base case $\eta=0.05-0.21$. The staggered case shows less anisotropic turbulence below the hub in comparison to the base case, with approximately $90 \%$ of the $\eta$ values above 0.19 located at the top rotor tip.

The spanwise normal Reynolds stress, shown in figure 7.3, does not have the high stresses that $\overline{u^{\prime \prime} u^{\prime \prime}} / U^{2}$ conveyed. There is also no direct correlation to magnitude of $\overline{v^{\prime \prime} v^{\prime \prime}} / U^{2}$ and vertical height. When increasing the spacing in both directions, the stress 

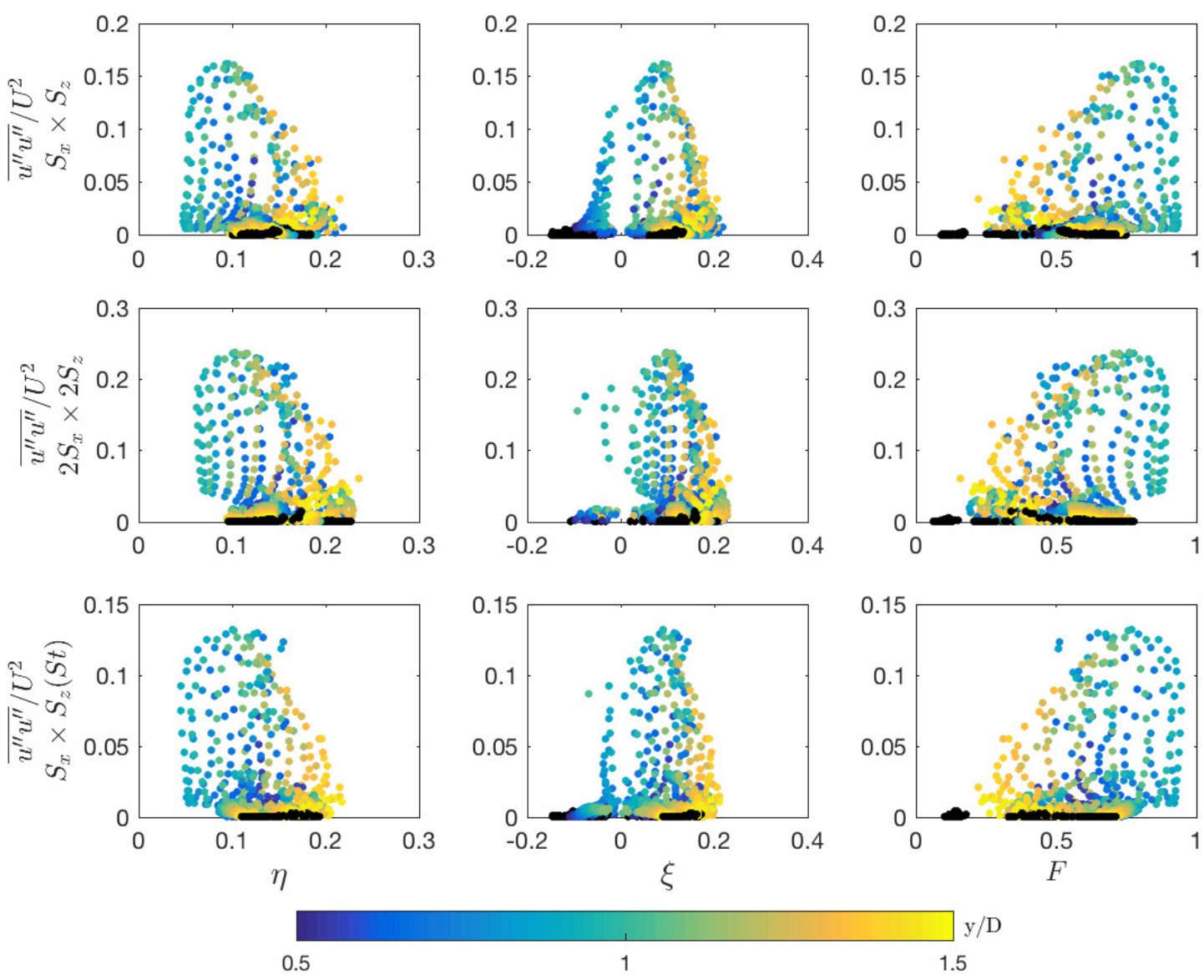

(A)

Figure 7.2: Dispersive normal streamwise stress vs. invariants in the near wake of the fully developed regime for $S_{x} \times S_{z}, 2 S_{x} \times 2 S_{z}, S_{x} \times S_{z}$ (St). The color map only includes the swept rotor area, whereas the black dots represent below the bottom rotor tip and above the rotor tip.

magnitude of $\overline{v^{\prime \prime} v^{\prime \prime}} / U^{2}$ increases to double that of the base case with outliers up to three times higher. This increase is not seen in the staggered case. For the staggered case all of the highest points of anisotropy occurs at the top rotor tip or at the bottom surface, whereas in the other two cases, there are points of high anisotropy near the top tip, bottom tip and hub. Most of the negative values for $\xi$ exist at the bottom surface 
with some points between the two tips. Most values for all three cases are positive indicating a dominance in stretched spheroid shapes. The case with increased spacing has less than half the amount of negative $\xi$ values as seen in the other two cases. The base case also has negative $\xi$ values for points at the top rotor tip where this is not present with the staggered case.
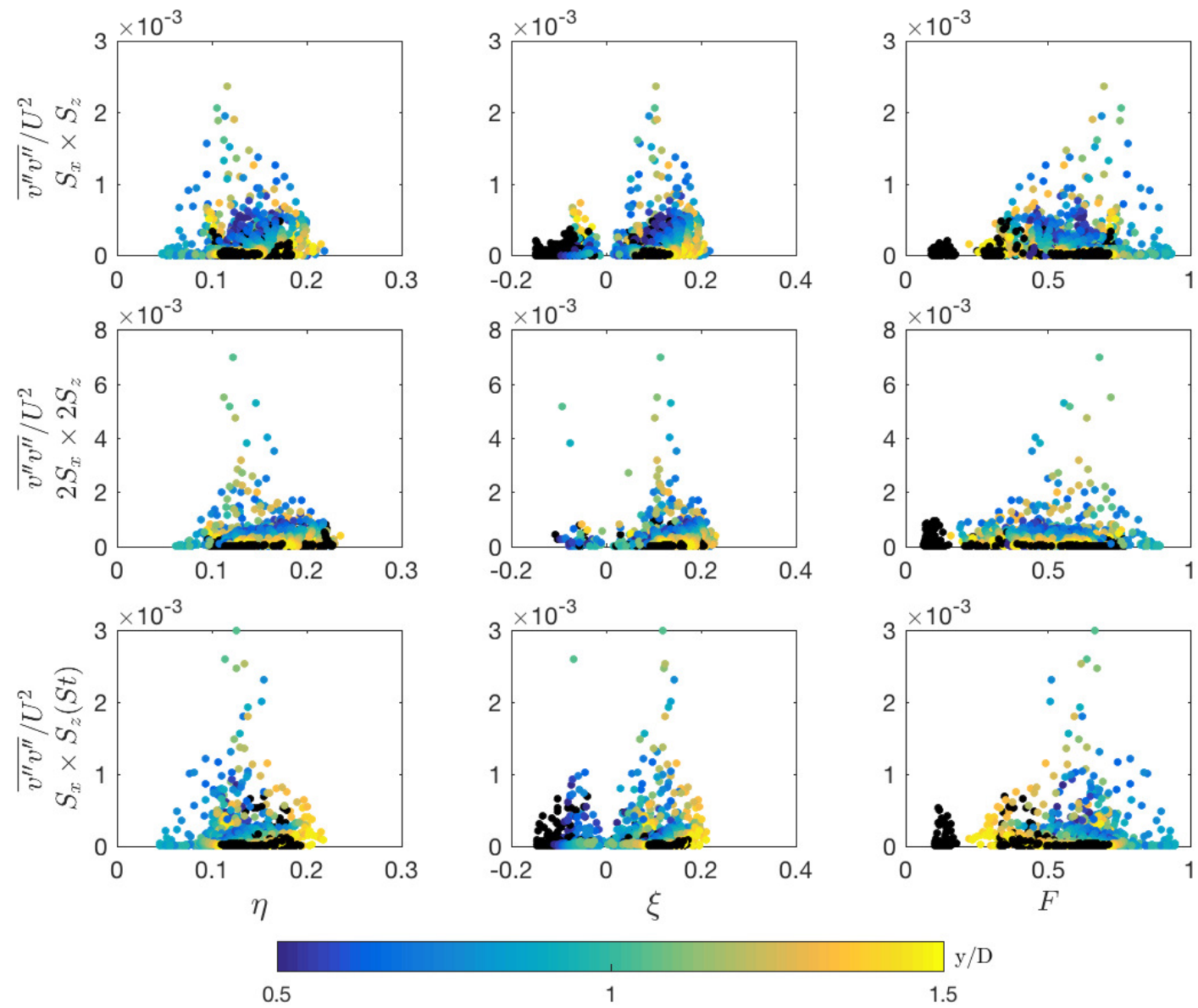

(A)

Figure 7.3: Dispersive normal spanwise stress vs. invariants in the near wake of the fully developed regime for $S_{x} \times S_{z}, 2 S_{x} \times 2 S_{z}, S_{x} \times S_{z}$ (St). The color map only includes the swept rotor area, whereas the black dots represent below the bottom rotor tip and above the rotor tip. 
The dispersive shear stress vs. invariants, shown in figure 7.4, also has a parabolic trend symmetric along the zero axis with the highest degrees of anisotropy close to the top rotor tip and bottom surface for all three cases. The highest magnitude of dispersive stresses occur between the hub and top rotor tip. When increasing the spacing in both directions, the magnitude of $-\overline{u^{\prime \prime} v^{\prime \prime}} / U^{2}$ doubles, but most of the points continue to lie close to the zero axis. The staggered case has similar $-\overline{u^{\prime \prime} v^{\prime \prime}} / U^{2}$ values to the base case. By increasing the spacing, areas between the bottom rotor tip and hub shift toward anisotropic behavior. The values of $F$ for $2 S_{x} \times 2 S_{z}$ decreases toward anisotropy by $20 \%$ from the base case. Similar to the other two dispersive stresses, the negative values for $\xi$ exist at the bottom surface with some points above the top tip, but most values are positive. Case $2 S_{x} \times 2 S_{z}$ has the least amount of negative $\xi$ values compared with the other two cases. 

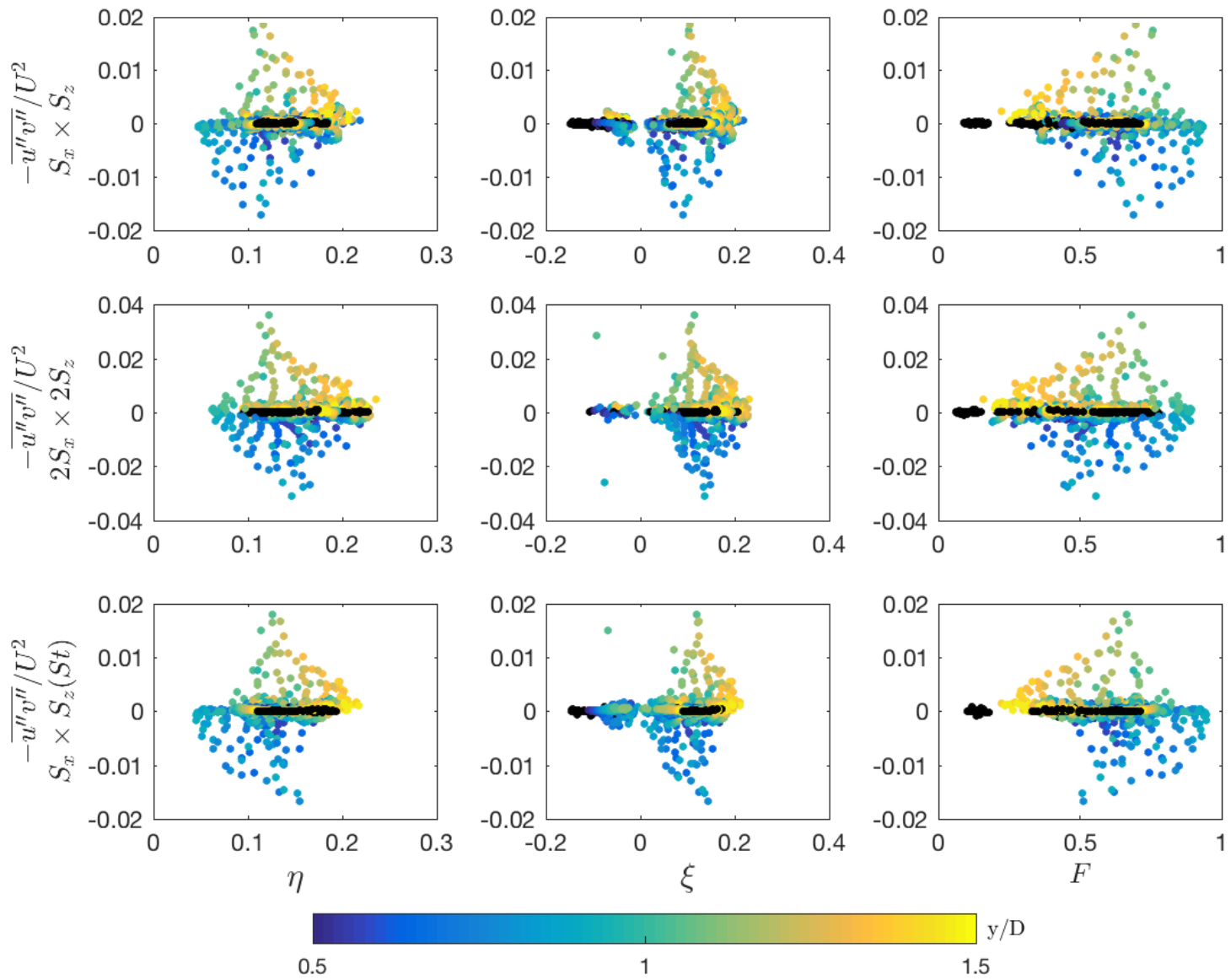

(A)

Figure 7.4: Dispersive shear stress vs. invariants in the near wake of the fully developed regime for $S_{x} \times S_{z}, 2 S_{x} \times 2 S_{z}, S_{x} \times S_{z}$ (St). The color map only includes the swept rotor area, whereas the black dots represent below the bottom rotor tip and above the rotor tip. Increased spacing results in an overall higher dispersive stress as well as an increase in anisotropy between the bottom rotor tip and hub. 


\section{Chapter 8}

\section{Conclusion}

Five LES turbine array cases with differences in streamwise and spanwise spacing were analyzed to see the relationship between Reynolds stress, dispersive stress and anisotropy. The densest cases $S_{x} \times S_{z}$ and $S_{x} \times S_{z}$ (St) experience the highest velocity deficit amid all five cases. Increasing the spanwise spacing results in high velocity channels in the streamwise direction between the turbines. The staggered case also shows the most rapid deceleration with magnitudes decreasing around $20 \%$ from the entrance to $x / D=32$.

The Reynolds shear stresses are the largest contributors to the mean kinetic energy budget, especially for flux and production. Cases $S_{x} \times S_{z}$ and $S_{x} \times 2 S_{z}$ both have the highest magnitude of Reynolds shear stress among the five cases, with stresses up to $14 \%$ larger. Increasing the streamwise spacing creates distinct breaks as the Reynolds shear stress diffuses between each row. Increasing the spanwise spacing correlates to an extension in $-\left\langle\overline{u^{\prime} v^{\prime}}\right\rangle / U^{2}$ stress channels downstream. TKE is highest further downstream in the domain and is particularly pronounced in the two most dense cases, $S_{x} \times S_{z}$ and $S_{x} \times S_{z}(S t)$.

For all cases, $\left\langle\overline{u^{\prime} u^{\prime}}\right\rangle / U^{2}$ and $\frac{\partial}{\partial y}\left\langle\overline{\left\langle u^{\prime} v^{\prime}\right.}\right\rangle / U^{2}$ are the highest contributor to the overall stress, and the most responsible for TKE production and kinetic energy flux. The nor- 
mal streamwise Reynolds stress is dominant near the bottom surface and top rotor tip due to the flow being forced in the streamwise $(u)$ direction while shearing with both the bottom surface and top tip. The denser canopies, $S_{x} \times S_{z}$ and (St), have the lowest Reynolds stress, $\left\langle\overline{u^{\prime} u^{\prime}}\right\rangle / U^{2}$, below hub-height due to the turbines obstructing with the shearing at the bottom surface. At the top rotor tip, the two dense cases peak in stress because there are more turbines present for shearing in this location. The vertical derivative, $\frac{\partial}{\partial y}\left\langle\overline{u^{\prime} v^{\prime}}\right\rangle / U^{2}$, is largest in the swept rotor area because the turbines are entraining energy from above. As the flow moves from developing to relaxed subdomain, the wake increases causing the vertical gradient to propagate upward.

Dispersive stresses are approximately 60\% larger in the developing region which correspond to a similar study that found that larger dispersive stresses at the entry region of the canopy [21]. The high dispersive stresses in the developing region are a result of the relative high velocities in the entrance of the canopy. A large velocity difference is then caused by the onset of turbines. The difference between the high velocities at the entrance and the turbine wake regions and bottom surface results in a high dispersive stress. In past studies, dispersive stresses conveyed far more dominance in sparse canopies over dense canopies. More spacing means the flow resistance is small and inner velocities and spatial deviations are high. In a dense canopy the inner average velocity and spatial deviation are small which results in a reduced dispersive stress. Results in this study show that those expected trends were not consistent. Sparse canopies have the highest $\left\langle\overline{u^{\prime \prime} u^{\prime \prime}}\right\rangle / U^{2}$ and $-\left\langle\overline{\left\langle u^{\prime \prime} v^{\prime \prime}\right.}\right\rangle / U^{2}$ values in the fully developed and relaxed regions, but in the developing subdomain the most dense cases, $S_{x} \times S_{z}$ and (St) have the highest dispersive shear and normal streamwise stresses. This is because as the flow is developing the dispersive stresses in the dense cases decay at 
a faster rate then the sparse cases due to high velocity deficit that exists in the dense cases. In the fully developed and relaxed subdomains, increasing the spanwise spacing, $S_{x} \times 2 S_{z}$, resulted in the highest $\left\langle\overline{\left\langle u^{\prime \prime} u^{\prime \prime}\right.}\right\rangle / U^{2}$ and $-\left\langle\overline{u^{\prime \prime} v^{\prime \prime}}\right\rangle / U^{2}$ magnitudes. Looking at the wake, the dispersive stress in the far wake is negligible, while in the near wake it resembles trends shown in the fully developed and relaxed subdomains. This highlights how the near wake dictates the dispersive stress for the flow.

Sparser canopies are related to higher anisotropy as well as larger dispersive stresses. Increasing the spacing between turbines in the spanwise, $2 S_{x} \times 2 S_{z}$ and $S_{x} \times 2 S_{z}$, reveal higher amounts of anisotropy below the top tip. At the top tip, an increase in streamwise spacing, $2 S_{x} \times 2 S_{z}$ and $2 S_{x} \times S_{z}$, reveals higher anisotropy. The denser cases, $S_{x} \times S_{z}$ and $S_{x} \times S_{z}(S t)$ exhibit the most isotropic behavior. This is due to the hub acting as a blunt body and disorganizing the flow. With more spacing between the turbines, the flow is able to recover and become more organized with more anisotropic turbulence. The most anisotropy turbulence is found at the bottom surface and at the top tip. The surface to bottom rotor tip between $y / D=0$ and $y / D=0.5$, is heavily affected by the ground surface roughness and near surface friction velocity, so it is expected to have high Reynolds stress levels as well as high anisotropy. The top tip also has high Reynolds stresses and anisotropy because the wind is shearing with the tip as it flows above it. There is a linear correlation between Reynolds stress and anisotropy. As Reynolds stress increases, so does anisotropy. The correlation is not as obvious with the dispersive stresses. The highest dispersive stress outliers gravitate toward isotropy, but most dispersive stresses lie midway on the spectrum. By increasing the spacing in both directions, the anisotropy of the dispersive normal streamwise stresses increases. The dispersive shear stress anisotropy of $2 S_{x} \times 2 S_{z}$ also increases 
between the bottom rotor tip and hub. Staggering the turbines results in a decrease in dispersive normal streamwise stress. 


\section{Chapter 9}

\section{Future Work}

Continuous exploration on the relationship between dispersive stress and anisotropy is attended. Studies include conducting an experiment with similar turbine array configurations to see if similarities exist or if there is more information on the relationships to be discovered. Another intention is to explore more configurations to provide even more insight on dispersive stress and anisotropy. Increasing the spacing even more is one possibility. More information allows for the development of models for the closure problems that exist in turbulence. Further insight on dispersive stress and anisotropy can help with the development of future wind turbines as well as providing more information on canopies themselves. 


\section{Bibliography}

[1] T. ACKermann, Wind power in power systems, John Wiley \& Sons, 2005.

[2] N. Ali, N. Hamilton, G. Cortina, M. Calaf, ANd R. B. Cal, Turbulence characteristics of a thermally stratified wind turbine array boundary layer: Part $i$, proper orthogonal decomposition, (2016).

[3] — Turbulence characteristics of a thermally stratified wind turbine array boundary layer: Part ii, anisotropy stress invariants, (2016).

[4] A. J. Brand, J. Peinke, AND J. Mann, Turbulence and wind turbines, in Journal of Physics: Conference Series, vol. 318, IOP Publishing, 2011, p. 072005.

[5] M. Breuer, S. RaY, V. Kumar, J. Jovanović, AND F. Durst, Anisotropy invariant reynolds stress model and its application to fully developed channel flows, PAMM, 5 (2005), pp. 537-538.

[6] M. Calaf, C. Meneveau, and J. Meyers, Large eddy simulation study of fully developed wind-turbine array boundary layers, Physics of fluids, 22 (2010), p. 015110.

[7] S. Cameron, V. Nikora, AND S. Coleman, Double-averaged velocity and stress distributions for hydraulically-smooth and transitionally-rough turbulent flows, Acta Geophysica, 56 (2008), pp. 642-653.

[8] O. Coceal, T. Thomas, I. Castro, And S. Belcher, Mean flow and turbulence statistics over groups of urban-like cubical obstacles, Boundary-Layer Meteorology, 121 (2006), pp. 491-519.

[9] M. EMORY AND G. IACCARINo, Visualizing turbulence anisotropy in the spatial domain with componentality contours.

[10] R. EsCudié AND A. Liné, Analysis of turbulence anisotropy in a mixing tank, Chemical engineering science, 61 (2006), pp. 2771-2779. 
[11] J. H. FERZIGER, Large eddy simulation: its role in turbulence research, in Theoretical approaches to turbulence, Springer, 1985, pp. 51-72.

[12] B. Frohnapfel, P. Lammers, J. Jovanović, And F. Durst, Interpretation of the mechanism associated with turbulent drag reduction in terms of anisotropy invariants, Journal of Fluid Mechanics, 577 (2007), pp. 457-466.

[13] D. Gielen, F. Boshell, AND D. SAYGIN, Climate and energy challenges for materials science, Nature materials, 15 (2016), pp. 117-120.

[14] N. HAmilton AND R. B. CAL, Anisotropy of the reynolds stress tensor in the wakes of wind turbine arrays in cartesian arrangements with counter-rotating rotors, Physics of Fluids (1994-present), 27 (2015), p. 015102.

[15] T. B. JOHANSSON AND L. BURNHAM, Renewable energy: sources for fuels and electricity, Island press, 1993.

[16] J. Jovanović, M. Pashtrapanska, B. Frohnapfel, F. Durst, J. Koskinen, And $\mathrm{K}$. KOSKINEN, On the mechanism responsible for turbulent drag reduction by dilute addition of high polymers: theory, experiments, simulations, and predictions, Journal of fluids engineering, 128 (2006), pp. 118-130.

[17] J. C. Kaimal AND J. J. Finnigan, Atmospheric boundary layer flows: their structure and measurement, Oxford university press, 1994.

[18] J. L. Lumley AND G. R. NEWMAn, The return to isotropy of homogeneous turbulence, Journal of Fluid Mechanics, 82 (1977), pp. 161-178.

[19] E. Martinot, A. Chaurey, D. Lew, J. R. Moreira, and N. Wamukonya, $R e-$ newable energy markets in developing countries, Annual review of energy and the environment, 27 (2002), pp. 309-348.

[20] J. Meyers and C. Meneveau, Optimal turbine spacing in fully developed wind farm boundary layers, Wind Energy, 15 (2012), pp. 305-317.

[21] S. Moltchanov, Y. Bohbot-Raviv, and U. Shavit, Dispersive stresses at the canopy upstream edge, Boundary-Layer Meteorology, 139 (2011), pp. 333-351.

[22] V. Nikora, D. Goring, I. McEwan, AND G. Griffiths, Spatially averaged open-channel flow over rough bed, Journal of Hydraulic Engineering, 127 (2001), pp. 123-133. 
[23] D. Poggi, G. Katul, And J. Albertson, A note on the contribution of dispersive fluxes to momentum transfer within canopies, Boundary-layer meteorology, 111 (2004), pp. 615-621.

[24] S. B. POPE, Turbulent flows, 2001.

[25] M. RAUPACH, P. COPPIN, AND B. LEGG, Experiments on scalar dispersion within a model plant canopy part $i$ : The turbulence structure, Boundary-Layer Meteorology, 35 (1986), pp. 21-52.

[26] M. Righetti And A. ARMAnini, Flow resistance in open channel flows with sparsely distributed bushes, Journal of Hydrology, 269 (2002), pp. 55-64.

[27] P. SADORSKY, Renewable energy consumption and income in emerging economies, Energy policy, 37 (2009), pp. 4021-4028.

[28] V. Sharma, G. Cortina, F. Margairaz, M. Parlange, and M. Calaf, Evolution of flow characteristics through finite-sized wind farms and influence of turbine arrangement, Renewable Energy, (2017).

[29] A. Simonsen And P.-Å. KRogstad, Turbulent stress invariant analysis: Clarification of existing terminology, Physics of Fluids, 17 (2005), p. 088103.

[30] J. SMAGORINSKY, General circulation experiments with the primitive equations: I. the basic experiment, Monthly weather review, 91 (1963), pp. 99-164.

[31] R. Smalley, S. Leonardi, R. Antonia, L. Djenidi, and P. Orlandi, Reynolds stress anisotropy of turbulent rough wall layers, Experiments in fluids, 33 (2002), pp. 31-37. 